\title{
Search for octupole-deformed actinium isotopes using resonance ionization spectroscopy
}

\author{
E. Verstraelen $\odot,{ }^{1, *}$ A. Teigelhöfer, ${ }^{2,3, \dagger}$ W. Ryssens, ${ }^{4}$ F. Ames, ${ }^{2}$ A. Barzakh, ${ }^{5}$ M. Bender,${ }^{6}$ R. Ferrer, ${ }^{1}$ S. Goriely, ${ }^{7,1}$ \\ P.-H. Heenen ${ }^{8,}$ M. Huyse, ${ }^{1}$ P. Kunz, ${ }^{2}$ J. Lassen,,${ }^{2,3, \dagger}$ V. Manea, ${ }^{1}$ S. Raeder, ${ }^{1,9}$ and P. Van Duppen ${ }^{1}$ \\ ${ }^{1}$ KU Leuven, Instituut voor Kern-en Stralingsfysica, Celestijnenlaan 200D, B-3001 Leuven, Belgium \\ ${ }^{2}$ TRIUMF, Canada's Particle Accelerator Centre, Vancouver, British Columbia V6T 2A3, Canada \\ ${ }^{3}$ University of Manitoba, Winnipeg, Manitoba R3T 2N2, Canada \\ ${ }^{4}$ Center for Theoretical Physics, Sloane Physics Laboratory, Yale University, New Haven, Connecticut 06520, USA \\ ${ }^{5}$ Petersburg Nuclear Physics Institute, NRC Kurchatov Institute, 188300 Gatchina, Russia \\ ${ }^{6} I P N L$, Université de Lyon, Université Lyon 1, CNRS/IN2P3, F-69622 Villeurbanne, France \\ ${ }^{7}$ Institut d'Astronomie et d'Astrophysique, CP229, Université Libre de Bruxelles, B-1050 Bruxelles, Belgium \\ ${ }^{8}$ PNTPM, CP229, Université Libre de Bruxelles, B-1050 Bruxelles, Belgium \\ ${ }^{9}$ GSI Helmholtzzentrum für Schwerionenforschung GmbH, 64291 Darmstadt, Germany
}

(Received 3 June 2019; published 28 October 2019)

\begin{abstract}
In-source resonance ionization spectroscopy of the neutron-rich actinium isotopes ${ }^{225-229} \mathrm{Ac}$ has been performed at the ISAC facility in TRIUMF, probing a ${ }^{2} D_{3 / 2} \rightarrow{ }^{4} P_{5 / 2}^{\circ}$ atomic transition. New data on the magnetic dipole moments and changes in mean-square charge radii $\delta\left\langle r^{2}\right\rangle$ of ${ }^{226,228,229} \mathrm{Ac}$ have been obtained. The comparison of the measured isotope shifts and magnetic dipole coupling constants $a\left({ }^{4} P_{5 / 2}^{\circ}\right)$ of ${ }^{225,227}$ Ac with a high-resolution data set is used to identify systematic uncertainties on the deduced $\delta\left\langle r^{2}\right\rangle^{A, 215}$ and magnetic dipole moment values. The charge radii odd-even staggering is evaluated for the odd- $N$ isotopes, showing that ${ }^{226} \mathrm{Ac}$ has an inverted odd-even staggering that might be linked with a reflection-asymmetric shape. Comparison of the magnetic dipole moments of ${ }^{225,227,229}$ Ac with Nilsson-model estimates supports the assumption of octupole deformation in isotopes ${ }^{225,227} \mathrm{Ac}$ and its gradual decrease toward isotope ${ }^{229} \mathrm{Ac}$. The changes in mean-square charge radii are compared to self-consistent calculations employing multiple modern energy density functionals: SLy5s1, BSk31, and DD-MEB1. For SLy5s1 in particular, self-consistent time-reversal breaking calculations of odd-odd nuclei incorporating finite octupole deformation are reported for the first time. For these calculations, the overall best agreement is obtained when the octupole degree of freedom is taken into account.
\end{abstract}

DOI: 10.1103/PhysRevC.100.044321

\section{INTRODUCTION}

The low-energy spectroscopy of nuclei unveils a large variety of phenomena. In the collective model, the gross features of many nuclear spectra can be explained by supposing that they are generated by deformed intrinsic states that can take different angular momenta in the laboratory frame. In the majority of cases, nuclear spectra can be understood by supposing that at low excitation energy the intrinsic states are axially and reflection symmetric. In some regions of the nuclear chart more general deformations have to be considered, such as octupole shapes.

Pear-shaped intrinsic configurations of nuclear ground states result from strong correlations between nucleons near the Fermi surface occupying single-particle states of opposite parity differing by three units of orbital and total angular momentum. This condition is fulfilled for proton or neutron numbers $Z, N \approx 34,56,88$, and 134 [1]. Their reflection asymme-

\footnotetext{
*elise.verstraelen@kuleuven.be

${ }^{\dagger}$ This article contains part of the experimental work performed during Teigelhöfer's Ph.D. thesis.

${ }^{\star}$ Deceased.
}

try manifests itself by the coexistence of levels of both parities in the spectra that are connected by strong dipole transitions. Most cases of indirect experimental indications for reflectionasymmetric intrinsic shapes (such as near-degenerate doublets of levels of the same angular momentum but opposite parity in odd-mass nuclei) have been observed in the actinium region $(Z \approx 88$ and $N \approx 134$ ), but some evidence in lighter nuclei exists as well, for example, in the barium region [2]. Nuclei with near-degenerate parity doublets offer a laboratory to study physics beyond the standard model that induces true breaking of reflection symmetry in the laboratory frame, thereby leading to a mixing of the states in the doublet [3].

First theoretical investigations of the appearance of octupole deformation were performed with the macroscopicmicroscopic model [4-6]. This approach had also been used as the starting point to construct particle-core coupling models to describe the low-energy spectroscopic properties of odd and odd-odd octupole deformed nuclei in the actinium region [6-10]. For even-even nuclei, the importance of octupole deformations in this mass region has been confirmed by HartreeFock plus Bardeen-Cooper-Schrieffer model (HF + BCS) and Hartree Fock Bogoliubov (HFB) calculations using different kinds of energy density functionals (EDFs) [11-14]. Beyondmean-field calculations using either a collective Hamiltonian 
based on the self-consistent solutions of constrained EDF calculations $[15,16]$, the symmetry-restored generator coordinate method (GCM) [17] or using an interacting-boson Hamiltonian with parameters determined by mapping the microscopic energy surfaces to the expectation value of the Hamiltonian in the boson condensate [18] are at present only available for even-even nuclei.

In the actinium region, the odd- $N$ neutron-rich radon, francium and radium isotopes exhibit larger charge radii than the average of their even- $N$ neighbors [19-23, and references therein]. This effect is usually referred to as inverted odd-even staggering, as in most other regions of the chart of nuclides isotopes with odd neutron number have a mean-square charge radius that is smaller than the mean of the values of adjacent isotopes with even $N$ [24]. In the actinium region, inverted odd-even staggering occurs exactly in the nuclei for which the presence of octupole deformation was proposed based on their nuclear spectroscopic properties [1,13]. It has been proposed that the underlying mechanism is the same as the one leading to the systematic difference of the splitting of parity doublets when comparing even-even, odd, and odd-odd nuclei. The analysis of such states in a schematic model indicates that the blocking of unpaired nucleons greatly reduces the fluctuations of the octupole moment, such that on the average low-lying states of nuclei with an unpaired neutron possess a larger octupole moment than states in the adjacent isotopes with even neutron number [6,19]. While the experimental observations are suggestive, the connection between octupole deformation and inverted odd-even staggering is not yet fully understood. For instance, the changes in mean-square charge radii of the neutron-rich francium and radium isotopes are reproduced by the extended Thomas-Fermi + Strutinski-Integral approximation to $\mathrm{HF}+\mathrm{BCS}$ with a Skyrme interaction without including the octupole degree of freedom [25].

In this article, the results of an in-source laser ionization spectroscopy study of ${ }^{225-229}$ Ac [26] performed at TRIUMF are reported and discussed along with the already-published isotopes ${ }^{212-215,225,227}$ Ac [27-30]. Laser-spectroscopy experiments provide information on the change in mean-square charge radii along an isotopic chain, the nuclear spin, and the electromagnetic moments via isotope-shift and hyperfinestructure measurements. The current measurements make it possible to investigate the odd-even staggering behavior and the trend of the magnetic dipole moments in the neutronrich actinium isotopes. The evolution of the mean-square charge radii and the magnetic dipole moments in the actinium isotopes, currently representing the heaviest isotopic chain crossing the $N=126$ shell closure, is discussed by presenting the TRIUMF data along with the previously measured results, which have not yet been described in former publications. The analysis of the experimental results will be complemented by an analysis of the role of octupole deformation for describing the mean-square charge radii within a state-of-the-art nuclear EDF calculation.

The paper is organized in the following way: Section II details the experimental technique and set-up; Sec. III A presents a description of the analysis of the hyperfine-structure spectra; Secs. III B and III C provide a comparison of the experimental results with data on other nuclei in the lead region;
Sec. IIID details the EDF calculations of the changes in mean-square charge radii; Sec. IIIE discusses the magnetic dipole moments; and Sec. IV summarizes the actinium laser spectroscopy results.

\section{EXPERIMENTAL SET-UP}

The actinium measurements were performed at three facilities using different laser resonance ionization spectroscopy methods while probing the same atomic transition. First, the neutron-deficient isotopes ${ }^{212-215}$ Ac were investigated using both on-line in-gas-cell (i.e., ${ }^{212-215} \mathrm{Ac}$ ) and in-gas-jet (i.e., ${ }^{214,215}$ Ac) laser spectroscopy at the LISOL facility in Louvainla-Neuve, Belgium [27,30]. Second, the neutron-rich isotopes ${ }^{225,227}$ Ac were studied using off-line perpendicular highresolution laser spectroscopy at the Johannes Gutenberg University in Mainz, Germany [28,33]. Finally, the neutron-rich isotopes ${ }^{225-229}$ Ac were measured at TRIUMF in Vancouver, Canada, using an ISAC on-line $\mathrm{UC}_{x}$ target after irradiation to perform in-source resonance ionization spectroscopy. The latter experimental set-up will be briefly described in the following. A detailed explanation can be found in Refs. [26,34]. For a description of the other two facilities, the reader is referred to Refs. [27,28,30,33].

The isotopes ${ }^{225-227} \mathrm{Ac}$ and ${ }^{225,227-229}$ Ac were measured at the ISAC facility [35] during two experimental campaigns in the years 2014 and 2016, respectively. The actinium isotopes were produced with a continuous beam of $480-\mathrm{MeV}$ protons with typically $10-\mu \mathrm{A}$ beam intensity irradiating a thick composite $\mathrm{UC}_{x}$ target [36]. Resonance ionization spectroscopy was conducted postirradiation on two targets that had received an integrated proton beam charge of $2515 \mu \mathrm{Ah}$ and $1755 \mu \mathrm{Ah}$, respectively. In order to reduce isobaric contamination of surface-ionized francium and radium isotopes, the measurements were performed directly after target irradiation, i.e., without proton beam on target. The isotopes produced inside the thick target diffused out of the target material and then effused inside the target container to make it into the 3-mm diameter $60-\mathrm{mm}$ long transfer/ionizer tube of the ISAC target/ion-source assembly. The mean time required for the actinium atoms to leave the target material and diffuse into the $3-\mathrm{mm}$ transfer tube was determined to be $\tau_{\text {release }}=20(6) \mathrm{h}$ at a target container heated up to $2000{ }^{\circ} \mathrm{C}$. Therefore only the long-lived actinium isotopes ${ }^{225} \mathrm{Ac}\left(T_{1 / 2}=10 \mathrm{~d}\right),{ }^{226} \mathrm{Ac}\left(T_{1 / 2}=29.37 \mathrm{~h}\right),{ }^{227} \mathrm{Ac}\left(T_{1 / 2}=\right.$ $21.77 \mathrm{y}),{ }^{228} \mathrm{Ac}\left(T_{1 / 2}=6.15 \mathrm{~h}\right)$, and ${ }^{229} \mathrm{Ac}\left(T_{1 / 2}=62.7 \mathrm{~min}\right)$ could be extracted from the $\mathrm{UC}_{x}$ target with significant yield. Inside the transfer/ionizer tube the atoms interacted with the laser light from the TRIUMF Resonant Ionization Laser Ion Source (TRILIS) and were ionized. The ionization scheme [31,37] (see Fig. 1) consisted of a 438.58-nm step to excite a ${ }^{2} D_{3 / 2} \rightarrow{ }^{4} P_{5 / 2}^{\circ}$ transition, which was scanned to probe the hyperfine structure (hfs) and isotope shift (IS), and a 424.69-nm step to ionize atoms via an autoionizing state. Laser wavelengths were measured with a wavemeter (High Finesse WS-7), which was calibrated monthly against a polarization stabilized He:Ne laser (Melles Griot 05-STP-901) that was in continuous operation. Both the 438.48- and 424.69-nm laser beams were produced by intracavity frequency doubling 


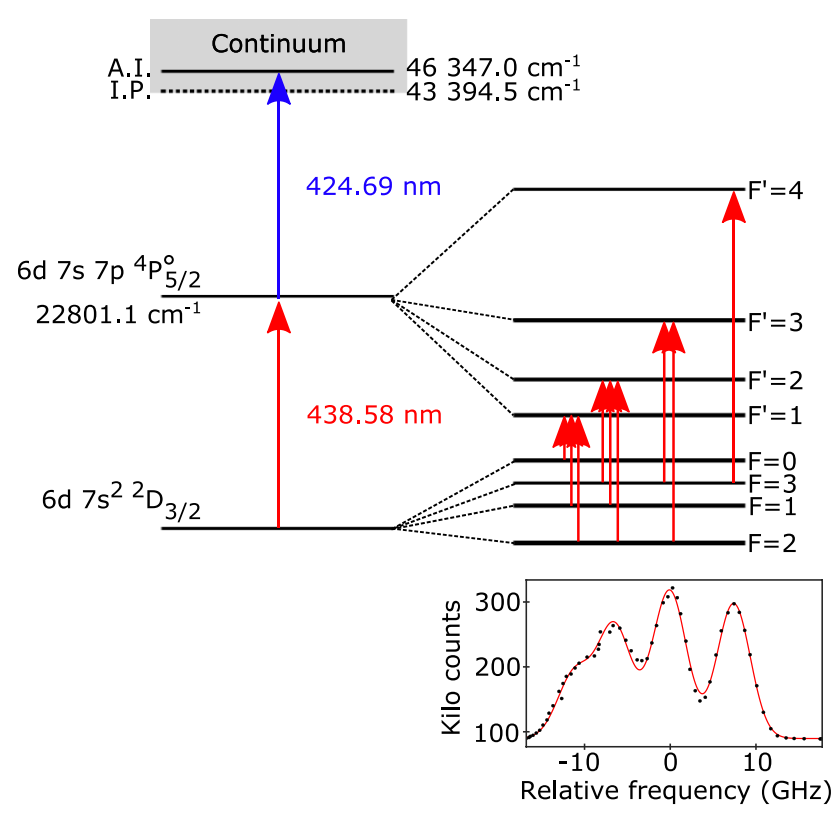

FIG. 1. Ionization scheme with vacuum wavelengths for actinium $[31,32]$. The right-hand side shows the hyperfine splitting for a $I=3 / 2$ nuclear spin (not to scale) together with a corresponding example hyperfine spectrum of ${ }^{227} \mathrm{Ac}$. The ${ }^{2} D_{3 / 2}$ splitting is unresolved, whereas the splitting of the ${ }^{4} P_{5 / 2}^{\circ}$ can be obtained from the distance between the peaks according to Eq. (2).

of two broadband Ti:Sa lasers with four-plate birefringent filters and $0.5-\mathrm{mm}$ solid etalons for wavelength selection and tuning. The obtained spectral linewidth of both lasers was $\sim 3 \mathrm{GHz}$. Each Ti:Sa laser was pumped with up to $10 \mathrm{~W}$ from a frequency-doubled Nd:YAG laser (LEE LPD $100 \mathrm{MQG)}$ at a repetition rate of $10 \mathrm{kHz}$. For intracavity frequency doubling either s-LBO or BBO crystals were used in angle-tuning configuration. The first-step laser was scanned by tilting a solid intracavity etalon with $40 \%$ reflectivity.

The laser beams were overlapped on the laser table via a polarizing beam-splitter (PBS) cube and transported via dielectric mirrors over a distance of $\sim 20 \mathrm{~m}$ into the ISAC target/ion source's ionizer tube. Individual laser beam intensities could be adjusted without beam displacement with a $\lambda / 2$ waveplate in front of the beam combining PBS. To reduce power broadening, the power of the first-step laser was reduced from the available hundreds of $\mathrm{mW}$ of UV light down to $5-25 \mu \mathrm{J} / \mathrm{cm}^{2}$. At the same time, the power of the second-step laser was reduced to $125-375 \mu \mathrm{J} / \mathrm{cm}^{2}$. Limited laser power fluctuations were present during a typical measuring time. Tests were performed to verify that the laser power did not drop due to detuning: A scan over the required frequency range was carried out while monitoring constantly the delivered power; no change in power was observed. After resonance ionization, the ions were extracted and accelerated to $20 \mathrm{keV}$. The extracted ion beam was subsequently separated according to the ion's mass to charge ratio $(A / q)$ by a twostage magnetic mass-separation system consisting of a preseparator and a high-resolution mass separator. All ions, except for ${ }^{226} \mathrm{Ac}$, were detected on a channeltron detector located $5 \mathrm{~m}$ downstream of the high-resolution mass separator. While the short-lived francium and radium isobars could be avoided in postirradiation in-source spectroscopy, at mass $A=226$, the long-lived surface-ionized ${ }^{226} \mathrm{Ra}\left(T_{1 / 2}=1600 \mathrm{y}\right)$ remained and required the use of the ISAC yield station [38] to identify the ${ }^{226} \mathrm{Ac}$ ion signal through $\beta$-decay detection on a set of scintillators resulting in a contamination-free measurement, as ${ }^{226} \mathrm{Ra}$ is a pure $\alpha$-emitter.

\section{RESULTS AND DISCUSSION}

\section{A. Fitting procedure}

Information on the magnetic dipole moment $\mu$ and the difference in mean-square charge radius $\delta\left\langle r^{2}\right\rangle^{A, A^{\prime}}$ between two isotopes with mass number $A$ and $A^{\prime}$ was obtained from the hyperfine spectra. The position of the hyperfine transitions is determined by [39]:

$$
v^{F, F^{\prime}}=v_{0}+\Delta v^{F^{\prime}}-\Delta v^{F},
$$

where $v_{0}$ denotes the center of gravity of the hfs, the prime symbol indicates the upper level and $\Delta v^{F}$ represents the frequency shift of the hyperfine component with total angular momentum $F$ with respect to the atomic level without hyperfine splitting and is equal to

$$
\Delta v^{F}=a \frac{K}{2}+b \frac{3 K(K+1)-4 I(I+1) J(J+1)}{8(2 I-1)(2 J-1) I J},
$$

where $K=F(F+1)-I(I+1)-J(J+1)$ with $I$ the nuclear spin and $J$ the atomic spin; $a$ and $b$ are the magnetic dipole and electric quadrupole hyperfine coupling constants, respectively. These constants are related to the nuclear magnetic dipole $(\mu)$ and spectroscopic quadrupole $\left(Q_{s}\right)$ moments by $a=\mu_{I} B_{e}(0) / I J$ and $b=e Q_{s} \frac{\partial^{2} V}{\partial z^{2}}$, where $B_{e}(0)$ and $\frac{\partial^{2} V}{\partial z^{2}}$ are the magnetic-field and electric-field gradient created by the electron cloud at the center of the nucleus, respectively.

The hyperfine-structure spectra were fit with the opensource python package SATLAS [40] using a $\chi^{2}$-minimization routine. As illustrated in Fig. 1 for ${ }^{227} \mathrm{Ac}$, the lower-state splitting of all isotopes was not resolved due to the Dopplerlimited resolution of the in-source spectroscopy method. Therefore, in the fitting procedure the ratios $a\left({ }^{4} P_{5 / 2}^{\circ}\right) / a\left({ }^{2} D_{3 / 2}\right)$ and $b\left({ }^{4} P_{5 / 2}^{\circ}\right) / b\left({ }^{2} D_{3 / 2}\right)$ were fixed to the literature values of ${ }^{227} \mathrm{Ac}$ [27], neglecting the hyperfine anomaly which is expected to be less than $1 \%$ [41]. The nuclear spins of ${ }^{225,227,228} \mathrm{Ac}$ were set equal to the literature assignments based on high-resolution laser-spectroscopy and beta-decay experiments (viz. $I=3 / 2, I=3 / 2$, and $I=3$, respectively) $[28,42]$. The nuclear spins of ${ }^{226,229}$ Ac were tentatively deduced from the upper-state splitting, which is resolved in the hyperfine spectra, since the number of hyperfine-structure peaks in our experiment correspond to the number of hyperfine sublevels of this upper state. The latter is equal to $2 I+1$ when $I<J=5 / 2$ and equal to $2 J+1=6$ when $I \geqslant$ $J=5 / 2$. Correspondingly, three hyperfine-structure peaks in the ${ }^{226} \mathrm{Ac}$ spectrum point to the $I=1$ assignment, whereas four peaks in the ${ }^{229} \mathrm{Ac}$ spectrum correspond to the $I=$ $3 / 2$ assignment (cf. also the similar shape of the hyperfinestructure spectra for ${ }^{225,227} \mathrm{Ac}$ with the well-established $I=3 / 2$ assignment). These tentative assignments are in 


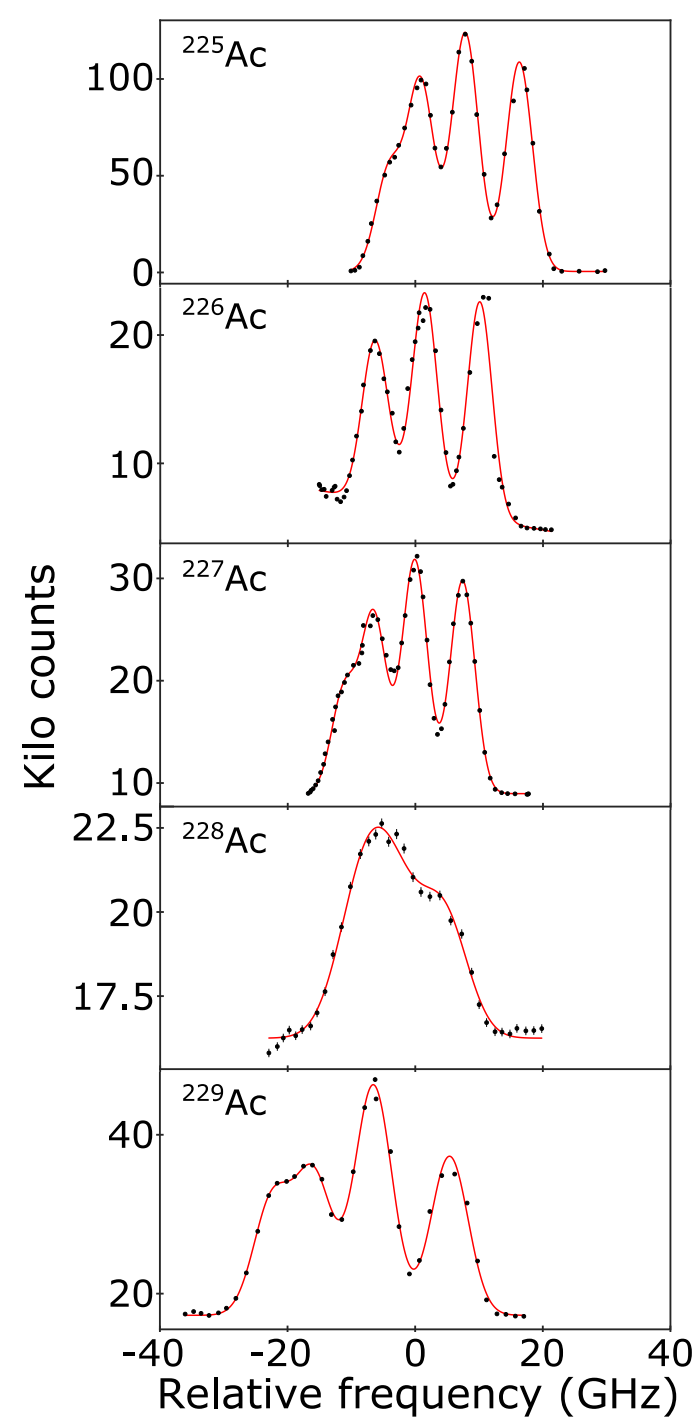

FIG. 2. Exemplary hyperfine spectra for all measured isotopes. The frequency is relative to the obtained center of gravity of ${ }^{227} \mathrm{Ac}$ $(683560 \mathrm{GHz})$. The fit to the data is represented by the solid lines.

agreement with the values suggested by $\beta$-decay experiments and transfer-reaction data [43,44]. The resonance line shapes were fully dominated by a Gaussian profile, originating from the Doppler broadening due to the high temperature of the source $\left(\sim 2000^{\circ} \mathrm{C}\right)$ as well as from the linewidth of the frequency-doubled 438.58-nm Ti:sapphire laser $(\sim 3 \sqrt{2} \mathrm{GHz})$. The experimental data were fit with the same full width at half maximum for all peaks. Within one unresolved multiplet, the intensity ratios were constraint to the theoretical Racah coefficients while the amplitudes of the multiplets were free fitting parameters. This dataanalysis approach resulted in the fits displayed in Fig. 2, which presents exemplary hyperfine spectra for all measured isotopes.

The multiple scans of each isotope were fitted separately resulting in values for the center of gravity and magnetic dipole coupling constant $a\left({ }^{4} P_{5 / 2}^{\circ}\right)$. The fit was sensitive neither to $a\left({ }^{2} D_{3 / 2}\right)$ nor to the $b\left({ }^{4} P_{5 / 2}^{\circ}\right)$ and $b\left({ }^{2} D_{3 / 2}\right)$ coupling constants. This was verified by explicitly changing the $a\left({ }^{4} P_{5 / 2}^{\circ}\right) / a\left({ }^{2} D_{3 / 2}\right)$ and or $b\left({ }^{4} P_{5 / 2}^{\circ}\right) / b\left({ }^{2} D_{3 / 2}\right)$ ratios. This resulted in the same centers of gravity and $a\left({ }^{4} P_{5 / 2}^{\circ}\right)$ values within uncertainties. As a final value for the center of gravity and magnetic dipole coupling constant $a\left({ }^{4} P_{5 / 2}^{\circ}\right)$, the weighted mean of the fit results was taken. To this weightedmean value both a statistical and systematic uncertainty was assigned. The statistical uncertainty is either the weighted average error or the weighted standard deviation depending on which value was larger. The systematic error, which includes indeterminacy of wavelength-meter calibration, was deduced by comparing the measured centers of gravity and magnetic dipole coupling constants $a\left({ }^{4} P_{5 / 2}^{\circ}\right)$ of ${ }^{225,227} \mathrm{Ac}$ that were measured within the corresponding experimental campaign with a high-resolution data set [28]. In the case of ${ }^{228} \mathrm{Ac}$, with unresolved hyperfine spectra (see Fig. 2), an additional systematic uncertainty was assigned by comparing to the weighted mean values obtained with different fit constraints on the quadrupole coupling constants: Quadrupole coupling constants fixed to zero and fixed to twice the literature values of ${ }^{227} \mathrm{Ac}$, which takes into account the spin difference between ${ }^{228} \mathrm{Ac}$ and ${ }^{227} \mathrm{Ac}$ according to the strong coupling scheme. The systematic uncertainty was set equal to the maximal deviation, including the statistical uncertainties.

\section{B. Changes in mean-square charge radii}

The isotope shifts were extracted from the fitted centers of gravity of the hyperfine-structure spectra compared to the reference isotope ${ }^{227} \mathrm{Ac}$. For consistency with the previously measured neutron-deficient actinium isotopes (Ref. [27]), the isotope shifts were converted relative to the same reference isotope, ${ }^{215} \mathrm{Ac}(N=126)$, using the literature isotope-shift value $\delta v^{227,215} \equiv v^{227}-v^{215}=-58319(10)\{133\} \mathrm{MHz}$ with the $1 \sigma$ statistical and systematic uncertainty given in parentheses and curly brackets, respectively [27].

The changes in the nuclear mean-square charge radii $\delta\left\langle r^{2}\right\rangle^{A, 215}$ were extracted from the measured isotope shifts $\delta v^{A, 215}$ via

$$
\delta\left\langle r^{2}\right\rangle^{A, 215}=\frac{\delta v^{A, 215}-M \frac{\left(m_{\mathrm{A}}-m_{215}\right)}{m_{\mathrm{A}} m_{215}}}{F},
$$

where $F$ is the field-shift and $M$ is the mass-shift constant, both depending on the atomic transition. The corresponding atomic parameters for the 438.58-nm transition in actinium were calculated in Ref. [27] using the multiconfiguration Dirac-Hartree-Fock method. The field-shift constant was found to be $F_{438.58}=-39(2) \mathrm{GHz} / \mathrm{fm}^{2}$ and the massshift constant $M_{438.58}=500(180) \mathrm{GHz} \cdot$ amu was obtained. This indicates a very small contribution of the mass shift to the total isotope shift (e.g., the mass shift of ${ }^{229} \mathrm{Ac}$ with respect to ${ }^{215} \mathrm{Ac}$ is equal to $142 \mathrm{MHz}$ ). The atomic masses $m_{\mathrm{A}}$ and $m_{215}$ were taken from Ref. [45]. The obtained $\delta\left\langle r^{2}\right\rangle^{A, 215}$ values along with their isotope shifts and assumed spins are shown in Table I. The literature values for ${ }^{225,227} \mathrm{Ac}[27,28]$ have also been included to provide a complete overview of the neutron-rich actinium region. The changes in the nuclear mean-square charge radii as a function of neutron number in 
TABLE I. Nuclear spins $I$, measured isotope shifts $\delta v^{A, 215}$ and deduced mean-square charge radii $\delta\left\langle r^{2}\right\rangle^{A, 215}$.

\begin{tabular}{|c|c|c|c|c|c|c|c|}
\hline $\begin{array}{l}\text { Mass } \\
\text { number }\end{array}$ & $\begin{array}{l}\text { Nuclear } \\
\text { spin } I^{\mathrm{a}}\end{array}$ & \multicolumn{2}{|c|}{ This work (2014) } & \multicolumn{2}{|c|}{ This work (2016) } & \multicolumn{2}{|c|}{ Previous work } \\
\hline 225 & $3 / 2^{\mathrm{d}}$ & $-50.3(0.06)\{0.2\}$ & $1.29(0.007)[0.07]$ & $-49.9(0.1)\{0.3\}$ & $1.28(0.01)[0.07]$ & $-50.2(0.02)\{0.1\}^{\mathrm{d}}$ & $\overline{1.29(0.003)[0.07]^{\mathrm{d}}}$ \\
\hline 227 & $3 / 2^{\mathrm{e}}$ & $\ldots$ & $\ldots$ & $\ldots$ & $\ldots$ & $-58.3(0.01)\{0.1\}^{\mathrm{e}}$ & $1.50(0.004)[0.08]$ \\
\hline 228 & $3^{f}$ & $\ldots$ & $\ldots$ & $-62(0.2)\{1\}$ & $1.59(0.03)[0.08]$ & $\ldots$ & $\ldots$ \\
\hline
\end{tabular}

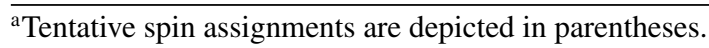

${ }^{\mathrm{b}}$ Statistical and systematic uncertainties are given in parentheses and curly brackets, respectively.

${ }^{\mathrm{c}}$ Total (statistical and systematic) experimental uncertainties are given in parentheses. The theoretical uncertainties, originating from the fieldand mass-shift values [27], are given in square brackets.

${ }^{\mathrm{d}}$ Ref. [28].

${ }^{\mathrm{e}}$ Ref. [27].

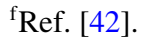

the lead-actinium region, relative to $N=126$, are displayed in Fig. 3.

The characteristic increase in the gradient of the $\delta\left\langle r^{2}\right\rangle^{N, 126}$ values beyond $N=126$, often referred to as a "kink," is assumed to be evident in the actinium isotopes. This shell effect in the mean-square charge radii was found to be a universal feature when crossing a neutron shell-closure [50] and has already been seen in i.a. lead [47], bismuth [48,50], polonium [51-53], and francium [57] in the vicinity of $N=$ 126. Understanding the occurrence of this kink in the meansquare charge radii at $N=126$ remains a challenge faced by theories of nuclear structure. For an extensive discussion on

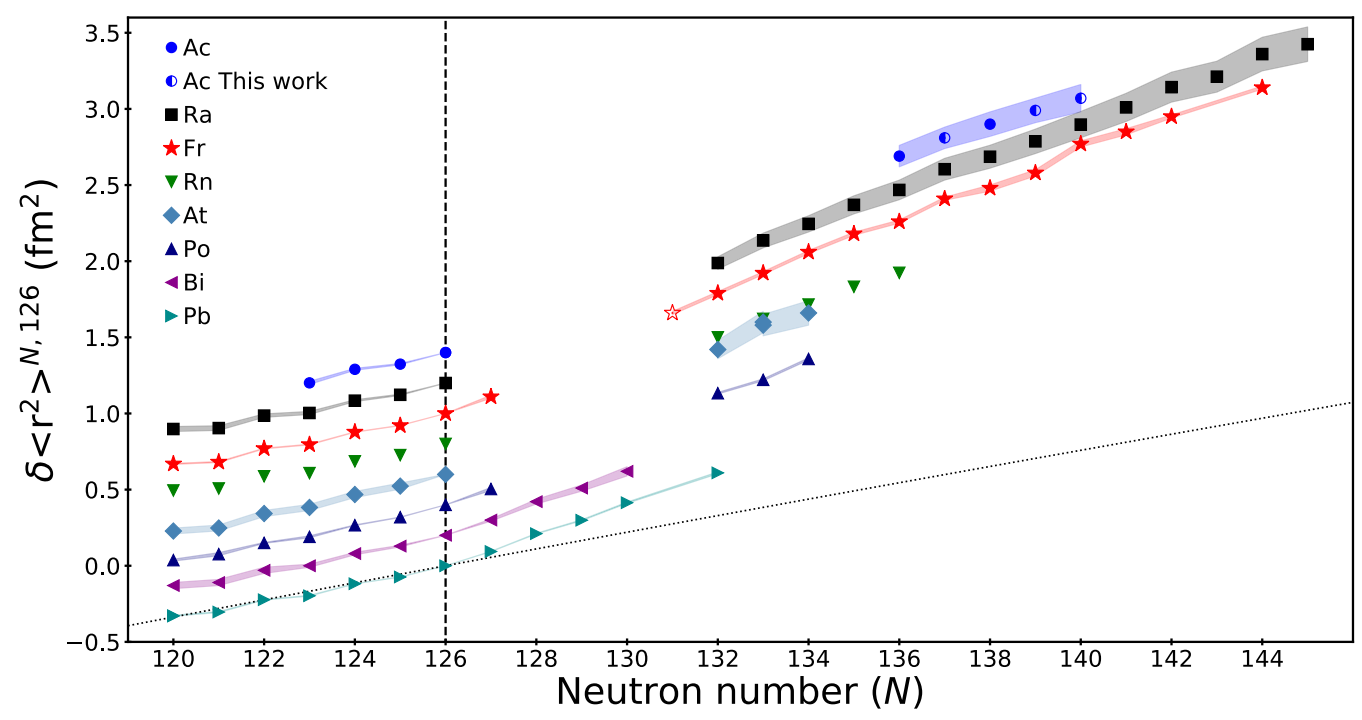

FIG. 3. $\delta\left\langle r^{2}\right\rangle^{N, 126}$ values in the lead-actinium region. An arbitrary offset of $0.2 \mathrm{fm}^{2}$ between the isotopic chains has been added for clarity. Dashed line: $N=126$ shell closure. Dotted line: Spherical droplet-model predictions for the lead isotopes [46]. Hollow symbols: Isomeric states. Shaded areas: Theoretical uncertainties originating from the calculation of the field- and mass-shift values. The experimental uncertainties, both statistical and systematic, are smaller than the symbol size. Right-pointing triangles: Pb, the isotope-shift values from Anselment et al. [47] have been re-evaluated using the reported atomic factors to separate the experimental and theoretical uncertainties of the $\delta\left\langle r^{2}\right\rangle^{N, 126}$ values. Left-pointing triangles: $\mathrm{Bi}$, the atomic factors reported by Barzakh et al. [48] have been used to deduce the $\delta\left\langle r^{2}\right\rangle^{N, 126}$ values from the isotope-shift data measured by Pearson et al. [49]; ${ }^{211} \mathrm{Bi}$ taken from Ref. [50]. Up-pointing triangles: Po, even isotopes [51]; ${ }^{217} \mathrm{Po}$ [52], the result for $I=9 / 2$ is presented; ${ }^{209,211} \mathrm{Po}$ [53], theoretical uncertainties of a magnitude similar to the experimental uncertainties have been considered; ${ }^{205,207} \mathrm{Po}$, the isotope-shift values deduced by Kowalewska et al. [54] have been converted to be with respect to ${ }^{210} \mathrm{Po}$. The converted isotope-shift values have been re-evaluated using the same approach as described by Cocolios et al. in Ref. [51]. Diamonds: At [55,56], all $\delta\left\langle r^{2}\right\rangle$ values were converted to be with respect to ${ }^{211}$ At. Down-pointing triangles: Rn [19], only experimental errors are reported. Stars: Fr, the isotope-shift values for ${ }^{207-213,220-228} \mathrm{Fr}$ [21] and ${ }^{218 m, 219,229,231} \mathrm{Fr}$ [22] have been re-evaluated using the quoted atomic factors to separate the experimental and theoretical uncertainties of the $\delta\left\langle r^{2}\right\rangle^{N, 126}$ values; ${ }^{214} \mathrm{Fr}$ taken from Ref. [57]. All $\delta\left\langle r^{2}\right\rangle$ values were converted to be with respect to ${ }^{213} \mathrm{Fr}$, using the results obtained by Dzuba et al. [21]. Squares: ${ }^{208-214,220-230,232} \mathrm{Ra}$ [23]; ${ }^{231,233} \mathrm{Ra}$ [58]. Circles: Ac, the isotope-shift values for ${ }^{212-215,227} \mathrm{Ac}$ [27] have been re-analysed using the cited $F$ - and $M$-values to separate the experimental and theoretical uncertainties of the $\delta\left\langle r^{2}\right\rangle^{N, 126}$ values; ${ }^{225} \mathrm{Ac}$ [28]. Left-filled circles: Ac, this work. The values for the neutron-rich actinium isotopes have been converted to be with respect to ${ }^{215} \mathrm{Ac}$ using the isotope-shift value $\delta v^{227,215}$ measured by Ferrer et al. [27]. 


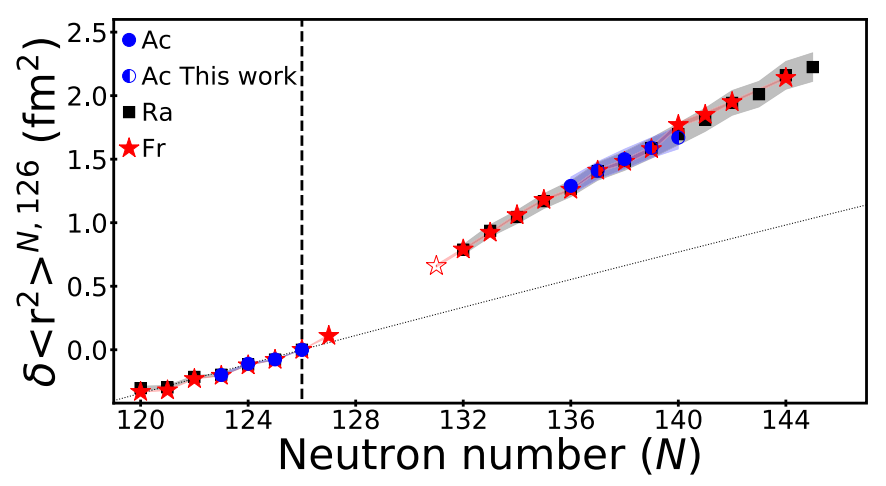

FIG. 4. Comparison of $\delta\left\langle r^{2}\right\rangle^{N, 126}$ values for francium (stars $[21,22,57])$, radium (black squares $[23,58]$ ), and actinium ((leftfilled) circles $[27,28])$. Dashed line: $N=126$ shell closure. Dotted line: Spherical droplet-model predictions for the actinium isotopes [46]. Hollow symbols: Isomeric states. Shaded areas: Theoretical uncertainties originating from the calculation of the field- and massshift values. The experimental uncertainties, both statistical and systematic, are smaller than the symbol size. A more detailed description can be found in the caption of Fig. 3 .

the different theoretical approaches to this shell effect the reader is referred to Refs. [50,57], and references therein. However, since no isotope shifts are available for the actinium isotopes immediately after the $N=126$ shell closure, due to their short life times, a more gradual gradient-transition or a kink at a higher neutron number cannot be excluded. Some theories predict this kind of behavior (see Sec. III D for more details).

Figure 4 shows the $\delta\left\langle r^{2}\right\rangle^{N, 126}$ values for the francium, radium, and actinium nuclei. Within the theoretical uncertainties, originating from the $F$ and $M$ factors, the isotopic dependencies of the $\delta\left\langle r^{2}\right\rangle^{N, 126}$ values are in agreement. The actinium isotopes follow the trend of the neighboring francium and radium isotopic chains on both the neutron-deficient and neutron-rich sides of the nuclear chart. A small deviation in the general trend of the francium isotopes is observed at $N=140$ signifying a change in odd-even staggering behavior with respect to the actinium and radium isotopic chains (see Fig. 5) [58].

\section{Odd-even staggering}

A well-known effect in the mean-square charge radii along an isotopic chain is the staggering between radii of odd- $N$ isotopes and their even- $N$ neighbors. This odd-even staggering effect can be quantified for odd- $N$ isotopes by the staggering parameter $\left(\gamma_{N}\right)$ introduced by Tomlinson and Stroke [59]:

$$
\gamma_{N}=\frac{\delta\left\langle r^{2}\right\rangle^{N-1, N}}{\frac{1}{2} \delta\left\langle r^{2}\right\rangle^{N-1, N+1}}
$$

which does not depend on the electronic factors and their large uncertainties.

Most nuclei exhibit normal odd-even staggering, i.e., $\gamma_{N}<$ 1 ; the mean-square charge radii of odd- $N$ nuclei is smaller than the average of their even- $N$ neighbors. This effect is often attributed to an interplay of quadrupole and pairing

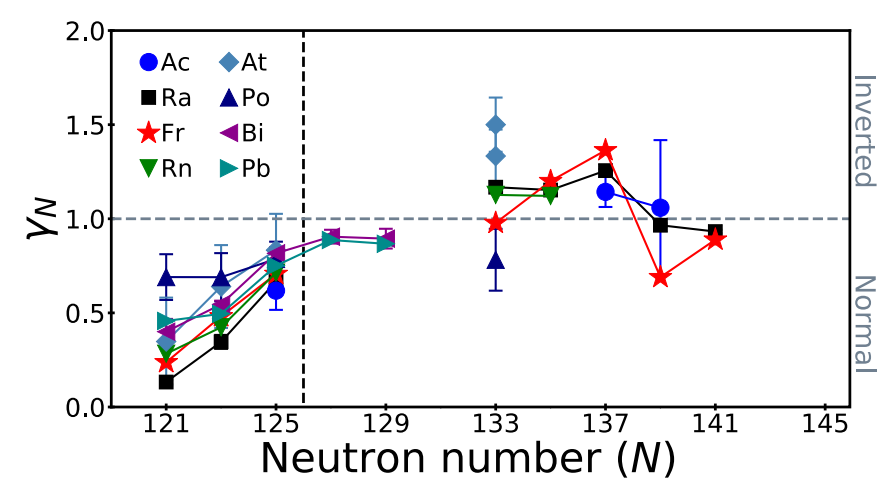

FIG. 5. Comparison of the odd-even staggering parameter $\gamma_{N}$ in the lead-actinium region evaluated from the mean-square charge radii displayed in Fig. 3. Dashed vertical line: $N=126$ shell closure. Dashed horizontal line: Absence of odd-even staggering, viz. $\gamma_{N}=$ 1. Circles: Ac [27,28]. Squares: Ra [23,58]. Stars: Fr [21,22,57]. Down-pointing triangles: Rn [19]. Diamonds: At [55,56]; the $\gamma_{N}$ value is shown for both $I\left({ }^{218} \mathrm{At}\right)=2$ and $I\left({ }^{218} \mathrm{At}\right)=3$. Up-pointing triangles: Po [51-54]. Left-pointing triangles: Bi [48-50]. Rightpointing triangles: $\mathrm{Pb}[47]$.

correlations. In this scenario, blocking of a neutron in an odd- $N$ isotope reduces the amplitude of quadrupole fluctuations compared to the adjacent even- $N$ isotopes, thereby slightly reducing the nuclear radius $[19,60]$. Leaving open its microscopic origin, the odd-even staggering of charge radii along isotopic chains of spherical nuclei has been successfully modeled in EDF calculations through an elaborate pairing energy functional with density-dependent and gradient terms proposed by Fayans et al. [61-63]. However, this type of pairing EDF has not been used to study the odd-even staggering of radii in symmetry-breaking calculations yet. An inversion of this staggering order $\left(\gamma_{N}>1\right)$, already identified in the astatine [56], radon [19], francium [22], and radium [58] isotopes around $N=135$, has been associated with reflection-asymmetric nuclear shapes $[1,60]$. Although the nature of this possible connection still remains an open question, a qualitative explanation can be found in terms of a polarization of an quadrupole-octupole deformed core toward a more stable octupole-deformed nucleus by the unpaired neutron $[1,22,60]$. However, inverted odd-even staggering has also been observed in regions of the nuclear chart which have not been linked to octupole deformation. In the light krypton and strontium region the observation of inverted odd-even staggering has been interpreted as an effect of polarization of the even-even core toward strong quadrupole deformation driven by the unpaired neutron [64]. Whereas the inverted staggering in the light mercury region has been explained as a quantum phase transition from a slightly quadrupoledeformed ground state toward a strongly deformed ground state when changing neutron number [65].

Figure 5 shows $\gamma_{N}$ as a function of neutron number in the lead-actinium region. Normal odd-even staggering is observed in the $N=121-129$ region of the nuclear chart with oddeven staggering parameters consistent for the wide range of proton numbers. The odd-even staggering parameter of ${ }^{226} \mathrm{Ac}$ is inverted and comparable to the values observed in the 
neutron-rich radium and radon isotopes. Due to the large experimental uncertainty, it can only be stated that ${ }^{228}$ Ac has a $\gamma_{N}$ value consistent with zero. Therefore no conclusions can be drawn on the disappearance of the inverted odd-even staggering as observed in francium at $N=139$.

\section{Comparison to EDF calculations}

As mentioned in the Introduction, approaches based on the microscopic-macroscopic method provide a rather satisfactory description of spectroscopic properties of even-even, odd, and odd-odd nuclei in this region when considering octupole deformation. However, charge radii obtained from such models are rarely discussed in the literature, exceptions being Refs. [66-68]. In that framework, calculating the charge radius from the macroscopic charge distribution entering the model systematically gives different values than calculating it from the single-particle wave functions [68], which introduces an ambiguity to the comparison with data. Compared to reflection-symmetric calculations, the overall size of the charge radii of radon, francium, and radium isotopes is better described when considering octupole deformation [67]. These authors point to a discrepancy between the deformations predicted by the model and the values deduced from in-band $B(E 2)$ values. However, it has to be noted that their analysis compares values obtained with two different definitions of deformation parameters that are not equivalent [10,69].

However, systematic reflection-symmetry-breaking calculations of nuclei with EDFs have been mostly limited to even-even nuclei. References [70,71] are notable exceptions that address a few selected odd-mass nuclei. The absence of such studies is related to the complexity, both technical and numerical, of treating blocked quasiparticles in that context. Their full treatment breaks the invariance of the HFB Hamiltonian under time reversal, which adds additional so-called "time-odd" terms to the EDF and increases the computational cost compared to calculations for even-even nuclei. An additional difficulty relates to selecting the correct quasiparticle configuration: In a self-consistent calculation the mean-fields are different for each, such that in general a full calculation has to be performed for several candidate configurations in order to identify the one giving the lowest energy.

In this section, the results of self-consistent EDF calculations for the entire actinium isotopic chain are presented for the first time and the calculated $\delta\left\langle r^{2}\right\rangle$ values are compared to the experimental results. Calculations have been carried out with three different EDFs: The DD-MEB2 [72] parametrization of a relativistic Lagrangian with density-dependent meson couplings, as well as the BSk31 [73] and SLy5s1 [74] parametrizations of the nonrelativistic Skyrme EDF. While SLy5s1 uses the standard form of the Skyrme EDF, BSk31 includes additional density dependencies of the gradient terms and corrections for various correlation effects.

\section{The parametrizations: DD-MEB2, BSk31, and SLy5s1}

The DD-MEB2 and BSk31 parametrizations on the one hand and the SLy5s1 parametrization on the other hand result from different readjustment philosophies. DD-MEB2 and BSk31 have both been adjusted as nuclear mass mod- els: Their parameters were determined primarily by fitting measured nuclear masses (with an rms deviation of 1.15 and $0.56 \mathrm{MeV}$, respectively, on all the 2353 measured masses of AME2012 [75]), properties of homogeneous neutron matter, as determined by many-body calculations with realistic twoand three-nucleon forces, as well as the charge-radii data with an rms deviation of 0.029 and $0.027 \mathrm{fm}$, respectively, on all the 884 measured values [76]. The parameters of SLy5s 1 were adjusted on experimental masses and charge radii of only a few doubly magic nuclei $\left({ }^{40} \mathrm{Ca},{ }^{48} \mathrm{Ca},{ }^{56} \mathrm{Ni},{ }^{132} \mathrm{Sn}\right.$, and ${ }^{208} \mathrm{~Pb}$ ) as well as some infinite nuclear matter properties and an additional constraint on the surface-energy coefficient [74]. It provides a satisfactory description of the deformation properties of very heavy nuclei, including the fission-barrier height of ${ }^{226} \mathrm{Ra}$ [69].

Aside from considerations about the relevant shape degrees of freedom, this collection of theoretical results allows us to investigate whether there is a systematic difference between the $\delta\left\langle r^{2}\right\rangle$ values predicted by relativistic and nonrelativistic EDFs in the region. Many relativistic approaches (although not all [77]) reproduce the "kink" in the radii of the lead isotopes rather well, whereas many traditional nonrelativistic parametrizations are known to be deficient in this respect. This success of the relativistic approach is often attributed to a specificity of its spin-orbit interaction [78]. Nonrelativistic approaches traditionally use a phenomenological ansatz for the two-body spin-orbit force with a single parameter. In relativistic approaches, a density-dependent spin-orbit field with a different isospin dependence arises as a natural consequence of Lorentz invariance without the need to posit its explicit form. However, in contrast to standard Skyrme or Gogny EDF most relativistic EDFs neglect the exchange terms. Doing the same in a nonrelativistic Skyrme EDF adds an isovector degree of freedom that can be exploited to reproduce the kink of charge radii at $N=126[79,80]$. The same generalized spin-orbit interaction is also used in the nonrelativistic Fayans functional that also quite successfully describes the charge radii around $N=126$ [61]. The converse is not true: Relativistic EDFs with exact exchange can still be adjusted to describe the kink [81]. It was, however, shown in Ref. [82] that a better description of the kink significantly deteriorates the description of the masses of the lead isotopes. It remains difficult to reproduce both the masses and the $\delta\left\langle r^{2}\right\rangle$ values accurately within one framework. It has been argued that the kink observed in the radii results from the (partial) occupation of the neutron $1 i_{11 / 2}$ orbital above $N=126$. The position of this orbital is influenced by many aspects of the EDF and not only by the details of the spin-orbit interaction.

It has to be noted that the parametrizations of the microscopic-macroscopic models for which charge radii have been analysed also do not reproduce the kink at $N=126$, as can be deduced from the change in slope of the deviation between theory and experiment as presented in Refs. [66,68].

\section{Conditions of the calculations}

None of our EDF solvers has the functionalities needed to treat all parametrizations considered here, and among them only one offers the possibility for symmetry-unrestricted 
calculations. As a consequence, the results will be grouped into two sets: one containing DD-MEB2 and BSk31 and the other one containing SLy5s1. Calculations with DD-MEB2 and BSk31 were performed with the harmonic-oscillatorbased solver used in Refs. [72,73]. For these two parametrizations, reflection and time-reversal symmetry, as well as axial symmetry, were assumed. The blocked quasiparticles are treated with the equal filling approximation, a numerically efficient approximation that, however, suppresses part of the polarization effects from the blocked nucleons [83]. The calculations discussed here reflect exactly the conditions during the original readjustments of these parametrizations.

The calculations with SLy5s1 on the other hand were carried out with the MOCCa code, that represents the wave functions in three-dimensional (3D) coordinate space. The code allows for the simultaneous breaking of reflection and time-reversal symmetry, and is equipped with algorithms that enable it to reliably find the one- or two-quasiparticle configuration with the lowest energy [84], and alleviates the numerical burden of symmetry-broken 3D calculations [85]. To assess the effect of octupole deformation, results obtained with SLy5s1 from full calculations allowing for reflectionasymmetric shapes and from restricted calculations that impose reflection symmetry are presented. The effects from time-reversal breaking from the blocked nucleons are always taken into account.

An additional difference between both sets of parametrizations is the calculation of charge radii. For DD-MEB2 and BSk31, the finite size of the proton and the intrinsic charge distribution of the neutron are taken into account through folding with suitable Gaussian form factors [86]. For SLy5s1, charge radii are directly calculated from the point proton density as the analytical correction for the finite size of the proton that are applied in the 3D codes [87] cancels out when calculating differences of mean-square radii.

\section{Multipole moments}

In order to characterize the shape of the nuclear density, the dimensionless multipole moments $\beta_{\ell m}$ [87] are used:

$$
\beta_{\ell m}=\frac{4 \pi}{3 R^{\ell} A}\left\langle\hat{r}^{\ell} \hat{Y}_{\ell m}(\theta, \phi)\right\rangle,
$$

where $\hat{Y}_{\ell m}$ is a spherical harmonic and $R=1.2 A^{1 / 3}$. These quantities are directly related to electromagnetic transition operators. They have to be distinguished from the deformation parameters $\beta_{\mathrm{WS}, \ell}$ of the expansion of the radius of the nuclear surface as used in microscopic-macroscopic models. Reference [10] provides the formulas that establish the relation between both types of deformation.

The possible values of the multipole moments are restricted by symmetries of the nuclear density: For reflectionsymmetric shapes $\beta_{\ell m}=0$ for odd values of $\ell$, while for axially symmetric shapes $\beta_{\ell m}=0$ for $m \neq 0$. In the calculations with SLy5s1, nonaxial degrees of freedom were explored, but all mean-field minima were found to be axially symmetric. The calculations with DD-MEB2 and BSk31 were restricted to axially symmetric configurations.

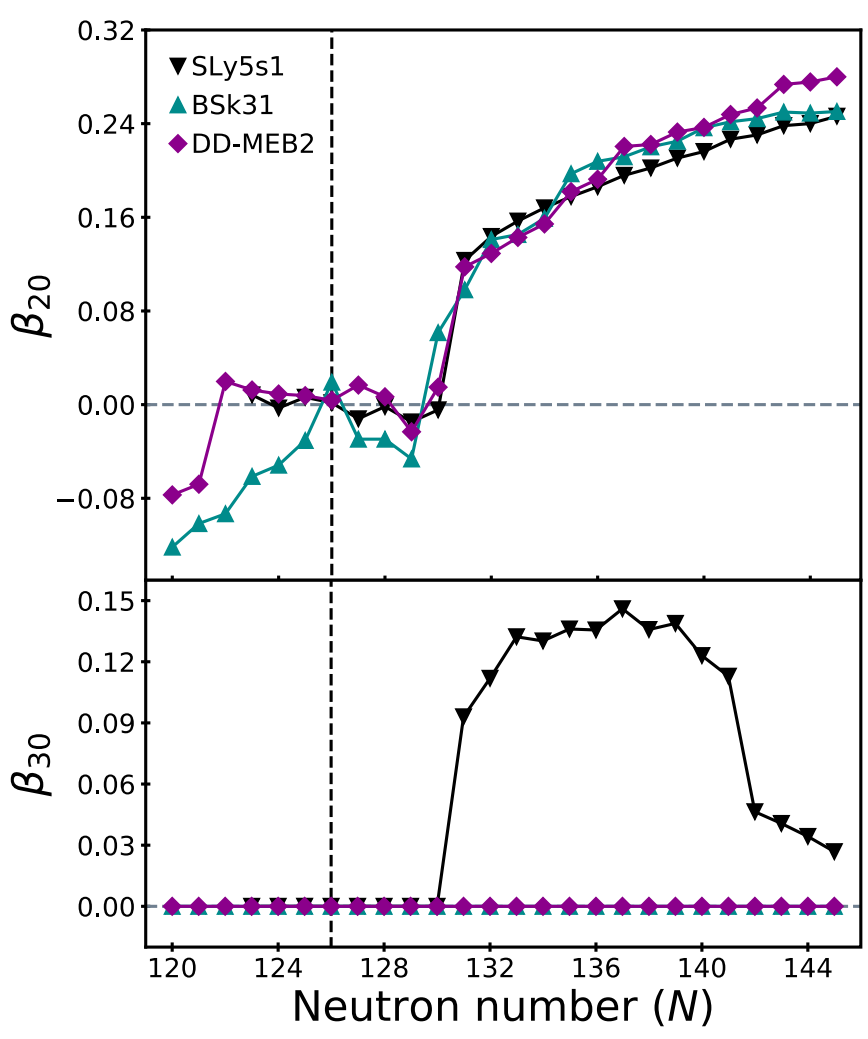

FIG. 6. Comparison of the $\beta_{20}$ and $\beta_{30}$ values for the SLy5s1, BSk31, and DD-MEB2 interactions. In the calculations with BSk31 and DD-MEB2 $\beta_{30}$ is restricted to 0 by the imposed reflection symmetry. Triangles: Nonrelativistic EDF values. Squares: Relativistic EDF values. Dashed line: $N=126$ shell closure.

In self-consistent calculations of a nucleus' ground state, all multipole moments not constrained to zero by symmetries automatically take a value that minimizes the energy. In case of the actinium isotopes, $\beta_{\ell m}$ up to $\ell=10$ take values that are significantly different from zero. For the discussion of radii, $\beta_{20}$ and $\beta_{30}$ are the dominant deformation modes and the discussion will be limited to their optimal values.

The role of the deformation of the nuclear density on the $\delta\left\langle r^{2}\right\rangle$ values can be easily understood in terms of a liquid-drop model: With increasing quadrupole deformation, the meansquare charge radius increases. When octupole deformation is added while keeping the quadrupole moment constant, the mean-square charge radius increases further. It has been argued in the past (see Sec. I) that it is the presence of octupole deformation that inverts the odd-even staggering $[1,60]$.

\section{Results and discussion}

The calculated $\beta_{20}$ and $\beta_{30}$ values of the actinium isotopes are shown in Fig. 6 for all three EDFs. For the lighter $(N<126)$ actinium isotopes SLy5s1 and DD-MEB2 yield near-spherical shapes, while BSk31 predicts oblate deformation. As the neutron number increases, a sharp transition to prolate shapes appears at $N=130$ for BSk31 and at $N=131$ for SLy5s1 and DD-MEB2, which for SLy5s1 also coincides with the onset of octupole deformation. For $N=131$, all parametrizations roughly agree on a quadrupole 


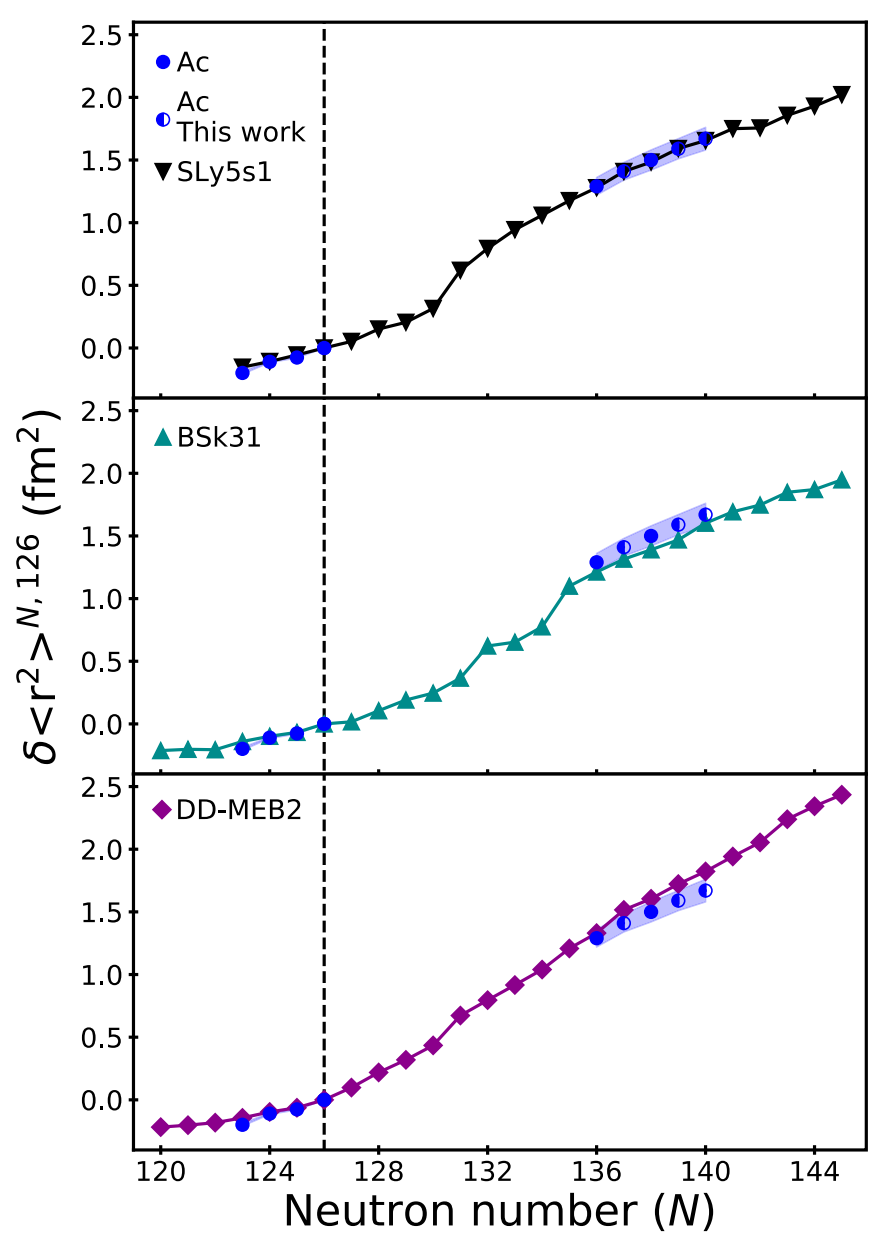

FIG. 7. Comparison of the $\delta\left\langle r^{2}\right\rangle^{N, 126}$ values for the SLy5s1, BSk31, and DD-MEB2 interactions. Triangles: Nonrelativistic EDF values. Squares: Relativistic EDF values. Dashed line: $N=126$ shell closure.

deformation of $\beta_{20} \simeq 0.12$, which then increases to about twice this value for $N=144$. For SLy5s 1 this increase is very gradual, whereas for the two others there is a second jump, at $N=135$ for BSk31 and at $N=143$ for DD-MEB2. For the heaviest isotopes, the latter then also gives slightly larger values than the other two parametrizations. The $\beta_{20}$ values obtained in symmetry-unrestricted calculations with SLy5s 1 of even-even isotopes of the adjacent thorium chain follow exactly the same trend and agree very well with the intrinsic charge quadrupole moment associated with $B(E 2)$ values in their ground-state bands [69].

Figure 7 shows the $\delta\left\langle r^{2}\right\rangle^{N, 126}$ values obtained using the different EDFs. All calculations reproduce the isotopic shifts for the light $(N \leqslant 126)$ actinium isotopes rather well, but differences arise for the heavier actinium isotopes. For isotopes right above the $N=126$ closure, the difference between relativistic and nonrelativistic parametrizations is the largest. Like for the lead isotopes, DD-MEB2 predicts a kink at $N=126$, whereas BSk31 and SLy5s1 do not. There is a consensus that this kink, like its counterparts at $N=28$ in the calcium chain [88,89] and at $N=82$ in the tin chain [90], is a phenomenon exhibited by spherical nuclei. Therefore,

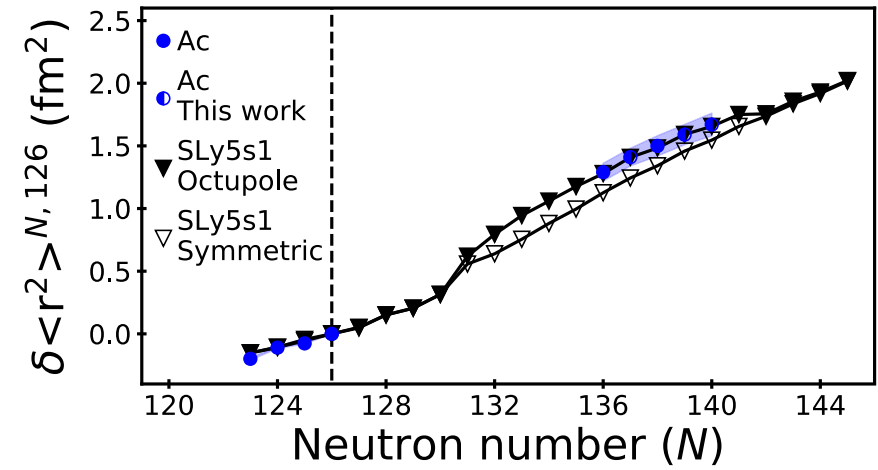

FIG. 8. Comparison of the experimental $\delta\left\langle r^{2}\right\rangle^{N, 126}$ values with calculations using the SLy5s1 interaction. Filled symboles (octupole): Allowing for reflection-asymmetric shapes. Hollow symbols (symmetric): Constrained to reflection-symmetric shapes. Dashed line: $N=126$ shell closure.

its correct description is not crucial for the discussion of the well-deformed actinium isotopes addressed here, although it remains an interesting open question how one phenomenon blends into the other. Because of the already enhanced radii of the near-spherical isotopes, the effect of the onset of deformation around $N=131$ on the isotopic shifts is much more gradual for DD-MEB2 than for the others. For SLy5s1, there is just one jump to larger values, whereas for BSk31 there are two, in each case for the same nuclei for which also the deformation changes abruptly. The SLy5s 1 results, allowing for octupole deformation, agree fairly well with the data, while BSk31 underestimates the experimental data but agrees with the slope of the experimental results. The relativistic DD-MEB2, despite its better description of the radii of the lead isotopes, overestimates the experimental results

To illustrate the effect of octupole deformation on the $\delta\left\langle r^{2}\right\rangle$ values, a set of calculations with SLy5s1 in which reflection-symmetry was enforced has also been performed. The $\delta\left\langle r^{2}\right\rangle^{N, 126}$ values obtained in this way, as compared to the reflection-asymmetric calculations, are presented in Fig. 8. The trend of quadrupole deformations is very similar in both calculations, and in particular for the isotopes ${ }^{225-229} \mathrm{Ac}$ the values are quasi-identical in both calculations. The SLy5s 1 calculations with reflection symmetry underestimate the experimental trend by about the same amount as BSk31, where reflection symmetry is always enforced.

The individual contributions from quadrupole and octupole deformation to the nuclear charge radius cannot be disentangled without looking also at other observables. In fact, there is no a priori reason that excludes the construction of an EDF that reproduces the $\delta\left\langle r^{2}\right\rangle$ values of ${ }^{225-229}$ Ac without considering octupole deformation. The $\beta_{20}$ values predicted by such an EDF would necessarily be larger than those obtained from a reflection symmetric SLy5s1 calculation. Such parametrization, however, would not be capable of describing other phenomena of nuclei in this region. First, it would overestimate the $\beta_{20}$ as inferred from $B(E 2)$ values in the neighboring even-even thorium isotopes. More importantly, it would be incapable of providing an explanation of the observation of parity-doublet bands in ${ }^{225} \mathrm{Ac},{ }^{227} \mathrm{Ac}$, and ${ }^{229} \mathrm{Ac}[10,60]$. 


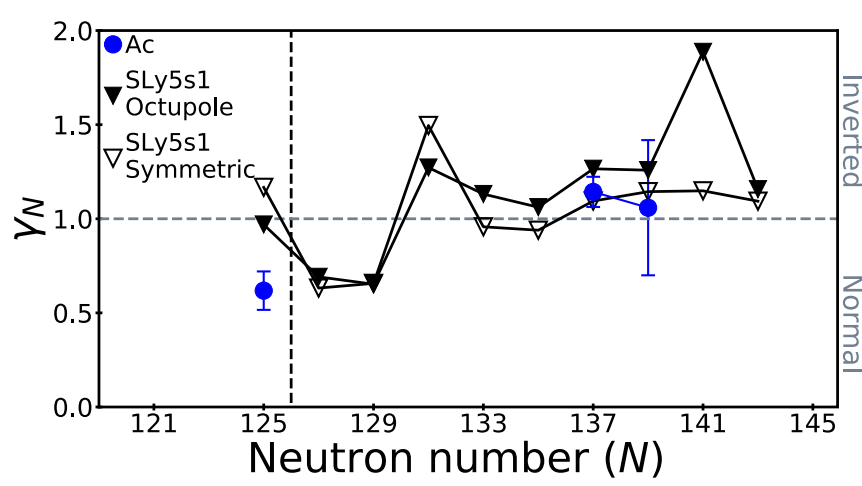

FIG. 9. Comparison of the experimental $\gamma_{N}$ values with calculations using the SLy5s1 interaction. Filled symboles (octupole): Allowing for reflection-asymmetric shapes. Hollow symbols (symmetric): Constrained to reflection-symmetric shapes. Vertical dashed line: $N=126$ shell closure. Horizontal dashed line: $\gamma_{N}=1$, marking no odd-even staggering.

A comparison between the odd-even staggering parameter $\gamma_{N}$ obtained with SLy5s1 in which reflection-symmetry was enforced, the reflection-asymmetric SLy5s1 calculations and the experimental values is presented in Fig. 9. The reflectionasymmetric SLy5s1 calculations predict an inverted odd-even staggering in the range of $N=131-143$, coinciding with the region of finite octupole deformation. When restricting the calculations to reflection-symmetric configurations, the inverted staggering disappears for $N=133$ and $N=135$ but persists for $N \geqslant 137$ albeit in a less pronounced fashion. Isotope ${ }^{220} \mathrm{Ac}(N=131)$ exhibits an inverted staggering in both calculations, which is directly related to the onset of deformation. The extreme inverted staggering for ${ }^{230} \mathrm{Ac}(N=$ 141) in the unrestricted calculation can also be directly linked to the disappearance of octupole deformation for $N=142$. These calculations hence suggest that the origin of inverted odd-even staggering in this region can at most be partially attributed to the role of octupole deformation: For several isotopes the inverted staggering persists even in the total absence of octupole deformation. A similar trend has been observed in the even-even radium isotopes in exploratory calculations [91].

\section{E. Magnetic dipole moments}

The nuclear magnetic moment $\mu_{A}$ of the actinium isotope with mass number $A$ was deduced from the following scaling relation:

$$
\mu_{A}=\mu_{215} \frac{I_{A}}{I_{215}} \frac{a_{A}\left({ }^{4} P_{5 / 2}^{\circ}\right)}{a_{215}\left({ }^{4} P_{5 / 2}^{\circ}\right)},
$$

where ${ }^{215} \mathrm{Ac}$ (with $I=9 / 2, g=0.920(13)$ and $a\left({ }^{4} P_{5 / 2}^{\circ}\right)=$ $2377(5)\{40\} \mathrm{MHz}[27,30])$ was used as a reference to be consistent with the normalization applied in Ref. [30] for the neutron-deficient actinium isotopes. In this relation the hyperfine anomaly between ${ }^{215} \mathrm{Ac}$ and the neutron-rich actinium isotopes is neglected. Usually, in this region of the nuclear chart the hyperfine anomaly is less than $1 \%$ (see Refs. [41,92] for the anomaly of the adjacent francium and ra-
TABLE II. Extracted hyperfine parameters $a\left({ }^{4} P_{5 / 2}^{\circ}\right)$.

\begin{tabular}{lccc}
\hline \hline Mass & This work $(2014)$ & This work $(2016)$ & Previous work \\
\cline { 2 - 4 } number & & $a\left({ }^{4} P_{5 / 2}^{\circ}\right)(\mathrm{MHz})^{\mathrm{a}}$ \\
\hline 225 & $2269(6)\{21\}$ & $2243(9)\{47\}$ & $2290(4)\{6\}^{\mathrm{b}}$ \\
226 & $2749(100)\{21\}$ & $\ldots$ & $\ldots$ \\
227 & $2084(31)\{21\}$ & $2176(27)\{71\}$ & $2105(3)\{5\}^{\mathrm{b}}$ \\
228 & $\ldots$ & $926(74)\{208\}$ & $\ldots$ \\
229 & $\ldots$ & $3142(37)\{71\}$ & $\ldots$ \\
\hline
\end{tabular}

${ }^{a}$ Statistical and systematic uncertainties are given in parentheses and curly brackets, respectively.

${ }^{\mathrm{b}}$ Ref. [28].

dium isotopes). Correspondingly, an additional $1 \%$ systematic uncertainty has been added to the magnetic dipole moments. Magnetic dipole coupling constants $a\left({ }^{4} P_{5 / 2}^{\circ}\right)$ and magnetic moments calculated using Eq. (6) are presented in Tables II and III, respectively.

$$
\text { 1. }{ }^{225,227,229} \mathrm{Ac}
$$

The magnetic dipole moment of a deformed odd-mass nucleus in the strong coupling scheme can be expressed as [93]:

$\mu_{\mathrm{odd}}=g_{R} I+\left(g_{K}-g_{R}\right) \frac{K^{2}}{I+1}\left(1+\delta_{K, 1 / 2}(2 I+1)(-1)^{I+1 / 2} b_{0}\right)$,

where $g_{K}$ is the intrinsic $g$ factor

$$
g_{K}=g_{l}+\frac{1}{K}\left(g_{s}-g_{l}\right)\left\langle s_{z}\right\rangle
$$

with $K$ the projection of the nuclear spin $I$ along the symmetry axis, $b_{0}$ the magnetic decoupling constant only relevant in the case of $K=1 / 2, g_{l}=g_{l}^{\text {free }}$ (with $g_{l p}=1$ and $g_{l n}=0$ [94]) and $g_{R}$ the rotational gyromagnetic ratio equal to $g_{R}=0.4$ and $g_{R}=0.25$ for odd- $Z$ and odd- $N$ nuclei, respectively [95]. Different renormalizations of $g_{s}$ factors have been considered in the actinium region. Reference [96] uses two options in the neighboring francium isotopes without a definite conclusion on which $g_{s}$ value has to be used in this region of the nuclear chart, viz. $g_{s}=0.6 g_{s}^{\text {free }}$ and $g_{s}=0.8 g_{s}^{\text {free }}$ with $g_{s p}^{\text {free }}=$ 5.587 and $g_{s n}^{\text {free }}=-3.826$ [94]. Whereas Refs. [6,97] take

TABLE III. Deduced magnetic dipole moments $\mu\left(\mu_{N}\right)$.

\begin{tabular}{lc}
\hline \hline Mass number & $\mu\left(\mu_{N}\right)^{\mathrm{a}}$ \\
\hline 225 & $1.33(0.019)\{0.04\}$ \\
226 & $1.06(0.04)\{0.03\}$ \\
227 & $1.22(0.018)\{0.03\}$ \\
228 & $1.1(0.09)\{0.3\}$ \\
229 & $1.82(0.03)\{0.07\}$ \\
\hline \hline
\end{tabular}

${ }^{a}$ Statistical and systematic uncertainties, including an $1 \%$ error due to the hyperfine-anomaly uncertainty, are given in parentheses and curly brackets, respectively. 


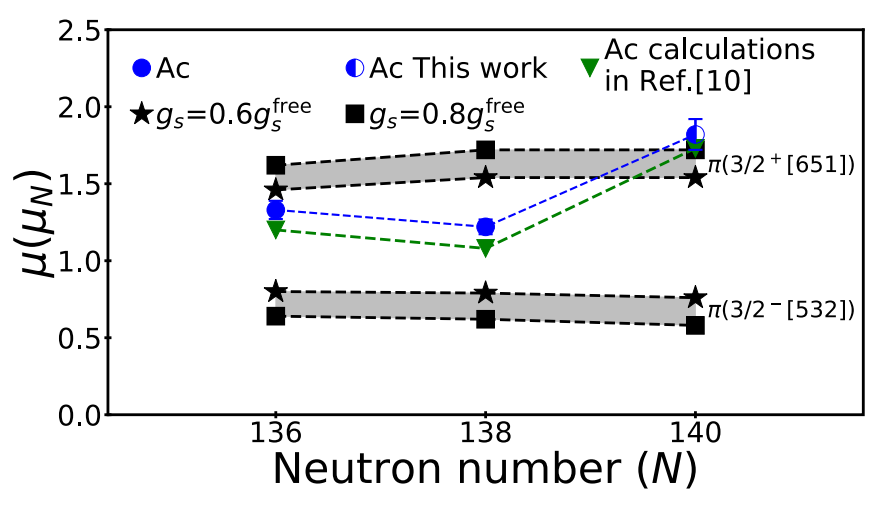

FIG. 10. Circles: Magnetic dipole moments for isotopes ${ }^{225,227} \mathrm{Ac}$ [28]. Left-filled circles: Magnetic dipole moment of isotope ${ }^{229} \mathrm{Ac}$ measured in this work. Error bars: Statistical and systematic uncertainties, including an $1 \%$ error due to the hyperfine-anomaly uncertainty. Down-pointing triangles: Theoretical calculations of the magnetic dipole moments of isotopes ${ }^{225,227,229}$ Ac performed by Leander and Chen in Ref. [10]. The theoretical magnetic dipole moments of the pure $\pi\left(3 / 2^{-}[532]\right)$ and $\pi\left(3 / 2^{+}[651]\right)$ configurations using $g_{s}=0.8 g_{s}^{\text {free }}$ and $g_{s}=0.6 g_{s}^{\text {free }}$ are shown in squares and stars, respectively. The shaded areas indicate the change of the theoretical magnetic moments with $g_{s}$ ranging from $0.6 g_{s}^{\text {free }}$ to $0.8 g_{s}^{\text {free }}$.

$g_{s}=0.7 g_{s}^{\text {free }}$. For $K=I \neq 1 / 2$, Eq. (7) simplifies to:

$$
\mu_{\text {odd }}=\frac{K}{K+1}\left(g_{R}+g_{K} K\right) .
$$

Isotopes ${ }^{225,227,229} \mathrm{Ac}$ have nuclear spin $I=3 / 2$. Near the proton Fermi level of actinium, there are two closely lying orbitals with $K=3 / 2: \pi\left(3 / 2^{-}[532]\right)$ and $\pi\left(3 / 2^{+}[651]\right)$. These spin-flipped states will strongly interact and will mix through an octupole field component resulting in a significant hybridization of the magnetic dipole moments.

Nilsson model calculations using a folded Yukawa potential have been performed by Leander and Sheline in Ref. [6] resulting in $\left\langle s_{z}\right\rangle$ values for the $\pi\left(3 / 2^{-}[532]\right)$ and $\pi\left(3 / 2^{+}[651]\right)$ orbitals in ${ }^{225,227,229}$ Ac. These $\left\langle s_{z}\right\rangle$ values have been determined at an equilibrium quadrupole deformation of $\beta_{\mathrm{WS}, 2} \approx 0.16$ (taken from Table I in Ref. [10]) under the constraint of reflection-symmetry. Here $\beta_{\mathrm{WS}, 2}$ is used instead of $\beta_{2}$ in the original work to emphasize the difference with the EDF $\beta_{20}$ values discussed in the previous sections. This $\beta_{\mathrm{WS}, 2}$ value is consistent with the EDF $\beta_{20}$ values from Sec. III D, since inserting $\beta_{\mathrm{WS}, 2}=0.169, \beta_{\mathrm{WS}, 3}=0.1$ and the $\beta_{\mathrm{WS}, 4}, \beta_{\mathrm{WS}, 5}$ and $\beta_{\mathrm{WS}, 6}$ values as listed in Ref. [10] in Eq. (2.26) herein for ${ }^{227} \mathrm{Ac}$, gives $\beta_{20}=0.202$. Using Eqs. (8) and (9), theoretical magnetic moments have been determined for the $\pi\left(3 / 2^{-}[532]\right)$ and $\pi\left(3 / 2^{+}[651]\right)$ configurations in $225,227,229 \mathrm{Ac}$ with $g_{s}$ ranging from $0.6 g_{s}^{\text {free }}$ to $0.8 g_{s}^{\text {free }}$. These theoretical values are displayed in Fig. 10 along with the experimental magnetic dipole moments of ${ }^{225,227,229} \mathrm{Ac}$.

The experimental magnetic dipole moments $\mu_{\exp }$ of ${ }^{225,227} \mathrm{Ac}$ point toward a mixing between the opposite-parity configurations $\pi\left(3 / 2^{-}[532]\right)$ and $\pi\left(3 / 2^{+}[651]\right)$ orbitals. In contrast, $\left.\mu_{\exp }{ }^{229} \mathrm{Ac}\right)$ agrees fairly well with the magnetic dipole moment of the pure $\pi\left(3 / 2^{+}[651]\right)$ state without in- voking octupole deformation and the opposite-parity mixing connected with it. This may indicate that ${ }^{229} \mathrm{Ac}$ lies at the borders of a region of octupole deformation, or at least this observation points to the decrease of $\beta_{\mathrm{WS}, 3}$ when going from ${ }^{227} \mathrm{Ac}$ to ${ }^{229} \mathrm{Ac}$. This trend in octupole deformation is supported by the change in energy splitting between the parity doublets in these actinium isotopes [98]. The EDF calculations also predict a decrease in octupole deformation in going from ${ }^{227} \mathrm{Ac}$ to ${ }^{229} \mathrm{Ac}$ and almost a disappearance after ${ }^{230} \mathrm{Ac}$ (see Fig. 6).

The magnetic dipole moments of ${ }^{225,227,229} \mathrm{Ac}$ have been calculated by Leander and Chen [10] using states of an octupole-deformed Wood-Saxon shell model coupled to a reflection-asymmetric rotor core. The best overall agreement between the theoretical and experimental spectroscopic properties was obtained at $\beta_{\mathrm{WS}, 3}=0.1$ and $\beta_{\mathrm{Ws}, 3}=0.07$ for ${ }^{225,227} \mathrm{Ac}$ and ${ }^{229} \mathrm{Ac}$, respectively. Figure 10 also shows the magnetic dipole moments of ${ }^{225,227,229} \mathrm{Ac}$ along with a comparison to the theoretical magnetic dipole moments obtained in Ref. [10] assuming $g_{s}=0.6 g_{s}^{\text {free }}$. The overall trend is reproduced. Note that in Ref. [10] ${ }^{229} \mathrm{Ac}$ is also considered to lie at the limits of the range of validity of the reflection-asymmetric rotor model since in order to match the experimental data Leander and Chen [10] were forced to decrease the $\beta_{\mathrm{WS}, 3}$ value in going from ${ }^{227} \mathrm{Ac}$ to ${ }^{229} \mathrm{Ac}$. This is in accordance with the EDF calculations and the qualitative analysis of the magnetic moment evolution mentioned above as well as with Möller et al. [99] calculations, who also predicted a noticeable decrease of octupolarity exactly at ${ }^{229} \mathrm{Ac}$. However, the theoretical values are systematically shifted toward smaller values. The experimental quadrupole moments of ${ }^{225,227} \mathrm{Ac}$ (1.55(0.05)[0.09] eb [28] and 1.74[0.1] eb [27], respectively, with the $6 \%$ systematic uncertainty stemming from atomic theory calculation [27]) are larger compared to Leander and Chen's values (1.21 eb and $1.44 \mathrm{eb}$, respectively). However, the increasing trend of the quadrupole moments in going from ${ }^{225} \mathrm{Ac}$ to ${ }^{227} \mathrm{Ac}$ is experimentally confirmed.

$$
\text { 2. }{ }^{228} \mathrm{Ac}
$$

The magnetic dipole moment of odd-odd nuclei can be calculated by the following relation [96]:

$$
\mu_{\text {odd-odd }}=\frac{K}{K+1}\left( \pm g_{K p} K_{p} \pm g_{K n} K_{n}+g_{R}\right)
$$

with

$$
K=\left|K_{p} \pm K_{n}\right|
$$

where $K_{i}$ is the projection of the angular momentum on the symmetry axis of the proton $(i=p)$ and neutron $(i=n)$ states, $g_{K}$ is the intrinsic $g$ factor and $g_{R}$ is the rotational gyromagnetic ratio $\left(g_{R}=Z / A \approx 0.4\right.$ is usually adopted for odd-odd nuclei in this region of the nuclear chart [10]). The signs in Eq. (10) are in accordance with the signs in Eq. (11). The intrinsic $g$ factors $g_{K i}$ can be calculated by Eq. (8). Alternatively, when $K \neq 1 / 2$, one can deduce empirical intrinsic $g$ factors $g_{K i \text {,emp }}$ from the measured magnetic dipole moments 
TABLE IV. Theoretical magnetic dipole moments of the pure $\pi\left(3 / 2^{-}[532]\right) \otimes v\left(3 / 2^{+}[631]\right)$ and $\pi\left(3 / 2^{+}[651]\right) \otimes v\left(3 / 2^{+}[631]\right)$ configurations at zero octupole deformation using $g_{s}=0.8 g_{s}^{\text {free }}$ and $g_{s}=0.6 g_{s}^{\text {free }}$. Compare with the experimental value $\mu_{\exp }\left({ }^{228} \mathrm{Ac}\right)=$ $1.1(0.09)\{0.3\} \mu_{N}$.

\begin{tabular}{lcc}
\hline \hline \multirow{2}{*}{ Configuration } & \multicolumn{2}{c}{$\mu_{\text {odd-odd,theo }}\left(\mu_{N}\right)$} \\
\cline { 2 - 3 } & $g_{s}=0.6 g_{s}^{\text {free }}$ & $g_{s}=0.8 g_{s}^{\text {free }}$ \\
\hline$\pi\left(3 / 2^{-}[532]\right) \otimes v\left(3 / 2^{+}[631]\right)$ & 0.76 & 0.48 \\
$\pi\left(3 / 2^{+}[651]\right) \otimes v\left(3 / 2^{+}[631]\right)$ & 1.70 & 1.85 \\
\hline \hline
\end{tabular}

$\mu_{\text {odd, exp }}$ [see Eq. (9)]:

$$
g_{K, \mathrm{emp}} K_{i}=\frac{K_{i}+1}{K_{i}} \mu_{\mathrm{odd}, \exp }-g_{R} .
$$

${ }^{228}$ Ac has $Z=89, N=139$, and $I=(3)$. For $Z=89$ and $\beta_{\mathrm{WS}, 3}=0$, the proton orbitals closest to the Fermi level are $\pi\left(3 / 2^{-}[532]\right)$ and $\pi\left(3 / 2^{+}[651]\right)$ (see discussion in Sec. III E 1). For $N=139$, the corresponding neutron orbital is $v\left(3 / 2^{+}[631]\right)$, which is the ground state of isotonic ${ }^{227} \mathrm{Ra}$ $(I=3 / 2)$ [6]. Using Eq. (8) with theoretical $\left\langle s_{z}\right\rangle$ values from Ref. [6] results in theoretical $g_{K}$ values of the $\pi\left(3 / 2^{-}\right.$[532]), $\pi\left(3 / 2^{+}[631]\right)$, and $v\left(3 / 2^{+}[631]\right)$ orbitals at zero octupole deformation with $g_{s}$ ranging from $0.6 g_{s}^{\text {free }}$ to $0.8 g_{s}^{\text {free }}$. By applying Eqs. (10) and (11), the theoretical magnetic dipole moments $\mu_{\text {odd-odd,theo }}$ of the $\pi\left(3 / 2^{-}[532]\right) \otimes v\left(3 / 2^{+}[631]\right)$ and $\pi\left(3 / 2^{+}[651]\right) \otimes v\left(3 / 2^{+}[631]\right)$ configurations at zero octupole deformation could be obtained (see Table IV). In this theoretical framework, $\mu_{\exp }\left({ }^{228} \mathrm{Ac}\right)=1.1(0.09)\{0.3\} \mu_{N}$ can be explained by a mixing between the spin-flipped $\pi\left(3 / 2^{-}[532]\right)$ and $\pi\left(3 / 2^{+}[651]\right)$ orbitals and therefore by including octupole deformation.

With $g_{K, \text { emp }}$ from adjacent odd- $A$ nuclei, calculated by Eq. (12) with the experimental magnetic dipole moments $\mu\left({ }^{227} \mathrm{Ac}\right)$ [28], $\mu\left({ }^{229} \mathrm{Ac}\right)$ (present work) and $\mu\left({ }^{227} \mathrm{Ra}\right)[60]$, one can determine $\mu_{\mathrm{emp}}$ for ${ }^{228} \mathrm{Ac}$ via Eq. (10): $\mu_{\mathrm{emp}}\left({ }^{228} \mathrm{Ac}\right.$; “227 $\left.\mathrm{Ac}+{ }^{227} \mathrm{Ra} "\right)=0.83(0.02)\{0.04\} \mu_{N}$ and $\mu_{\text {emp }}\left({ }^{228} \mathrm{Ac}\right.$; “229 Ac $\left.+{ }^{227} \mathrm{Ra} "\right)=1.58(0.04)\{0.09\} \mu_{N} . \quad$ Evidently, only with $\mu_{\mathrm{emp}}$ assuming the same proton and neutron states in ${ }^{228} \mathrm{Ac}$ as in ${ }^{227} \mathrm{Ac}$ and ${ }^{227} \mathrm{Ra}$, one can match $\mu_{\exp }\left({ }^{228} \mathrm{Ac}\right)=1.1(0.09)\{0.3\} \mu_{N}$. (Note that Möller et al. [99] predicted very close quadrupole and octupole deformation parameters for ${ }^{227} \mathrm{Ac}$ and ${ }^{227} \mathrm{Ra}$.) The experimental magnetic moment of ${ }^{227} \mathrm{Ac}$ (see Sec. IIIE 1) and ${ }^{227} \mathrm{Ra}[6,10]$ are explained in the framework of a single-particle approach with nonzero octupole deformation and, when used for the additivity rule, explain $\mu_{\exp }\left({ }^{228} \mathrm{Ac}\right.$ ) hinting to the presence of octupole deformation.

\section{3. ${ }^{226} \mathrm{Ac}$}

The spin of ${ }^{226} \mathrm{Ac}(Z=89$ and $N=137)$ has tentatively been assigned as $I=(1)$ (see Sec. III A). Assuming that the odd proton in ${ }^{226} \mathrm{Ac}$ remains in the same $K=3 / 2$ orbital as in the adjacent isotopes ${ }^{225,227} \mathrm{Ac}$, the neutron state has to be chosen with either $K=5 / 2$ or $K=1 / 2$ to match this spin assignment.
TABLE V. Empirical magnetic dipole moments for ${ }^{226} \mathrm{Ac}$ obtained by coupling the proton states of ${ }^{225,227} \mathrm{Ac}(I=3 / 2)$ with the neutron states of ${ }^{221,229} \mathrm{Ra}(I=5 / 2)$. Compare with the experimental value $\mu_{\exp }\left({ }^{226} \mathrm{Ac}\right)=1.06(0.04)\{0.03\} \mu_{N}$.

\begin{tabular}{lcc}
\hline \hline Proton state & Neutron state & $\mu_{\text {emp }}\left(\mu_{N}\right)$ \\
\hline${ }^{227} \mathrm{Ac}$ & ${ }^{221} \mathrm{Ra}$ & $-0.868(0.015)\{0.025\}$ \\
${ }^{225} \mathrm{Ac}$ & ${ }^{221} \mathrm{Ra}$ & $-0.959(0.016)\{0.033\}$ \\
${ }^{227} \mathrm{Ac}$ & ${ }^{229} \mathrm{Ra}$ & $-0.390(0.015)\{0.025\}$ \\
${ }^{225} \mathrm{Ac}$ & ${ }^{229} \mathrm{Ra}$ & $-0.482(0.016)\{0.033\}$ \\
\hline \hline
\end{tabular}

The ground states in ${ }^{221,229} \mathrm{Ra}$ have spin $I=5 / 2$ with the leading Nilsson configurations being $v\left(5 / 2^{+}[633]\right)$ and $v\left(5 / 2^{+}[622]\right)$, respectively [100]. Both Nilsson states are relatively close to the neutron Fermi level of ${ }^{226} \mathrm{Ac}$ and have to be considered as possible neutron states in ${ }^{226} \mathrm{Ac}$. Applying Eq. (12) with the experimental magnetic dipole moments of isotopes ${ }^{225,227} \mathrm{Ac}$ and ${ }^{221,229} \mathrm{Ra}$ [60], results in empirical $g_{K, \text { emp }}$ factors which can be inserted in Eq. (10) to obtain empirical magnetic dipole moments of ${ }^{226} \mathrm{Ac}$. These values are summarized in Table V. Neither of these combinations can reproduce the sign of $\mu_{\exp }\left({ }^{226} \mathrm{Ac}\right)=1.06(0.04)\{0.03\} \mu_{N}$.

Spin $I=1$ can also be obtained by coupling the $K=3 / 2$ proton states of ${ }^{225,227} \mathrm{Ac}$ with the $K=1 / 2$ neutron state of isotonic ${ }^{225} \mathrm{Ra}(I=1 / 2)$, which stems from the $v\left(1 / 2^{+}[631]\right)$ Nilsson orbital with an admixture of the opposite-parity states $v\left(1 / 2^{-}[770]\right)$ and $v\left(1 / 2^{-}[761]\right)$ due to octupolarity [101]. From Eq. (7) it follows that:

$$
g_{K} K=\frac{K+1}{K\left(1-2 b_{0}\right)} \mu+\left(K-\frac{K+1}{1-2 b_{0}}\right) g_{R}
$$

when $K=I=1 / 2$. Therefore, to extract $g_{K n \text {, emp }} K_{n}$ values from the measured $\mu_{\exp }\left({ }^{225} \mathrm{Ra}\right)=-0.7338(15) \mu_{N}$ [60], the magnetic decoupling factor $b_{0}$ of this ground-state configuration has to be known. The magnetic decoupling factor $b_{0}$ can be deduced from the experimentally determined rotational decoupling factor $a$ via the relation:

$$
\left(g_{K}-g_{R}\right) b_{0}=-\left(g_{l}-g_{R}\right) a-\frac{1}{2}(-1)^{l}\left(g_{s}+g_{K}-2 g_{l}\right) .
$$

By solving the set of Eqs. (13) and (14) with $\left.a\left({ }^{225} \mathrm{Ra}\right)=1.4[101], \quad \mu_{\exp }{ }^{225} \mathrm{Ra}\right)=-0.7338(15) \mu_{N}$ and $g_{s}=\{0.6,0.8\} g_{s, \text { free }}$, one obtains $b_{0}=\{-1.64,-2.64\}$ and $g_{K n, \text { emp }} K_{n}=\{-0.2855,-0.4768\}$, respectively. The $\mu_{\text {emp }}$ values obtained by coupling the proton states of ${ }^{225,227} \mathrm{Ac}$ with the neutron state of ${ }^{225} \mathrm{Ra}$ are presented in Table VI. $\mu_{\text {emp }}\left({ }^{(227} \mathrm{Ac}+{ }^{225} \mathrm{Ra} "\right)=1.159(0.015)\{0.025\} \mu_{N} \quad$ (taking $\left.g_{s}=0.8 g_{s, \text { free }}\right)$ agrees fairly well with the experimental result $\mu_{\exp }(226 \mathrm{Ac})=1.06(0.04)\{0.03\} \mu_{N}$. It should be noted that the magnetic moments of ${ }^{227} \mathrm{Ac}$ and ${ }^{225} \mathrm{Ra}$ can currently only be explained with the assumption of nonzero octupole deformation [10,101] and that Möller et al. predicts comparable quadrupole and octupole deformation parameters for both isotopes $[99,101]$. Similarly as for the case of ${ }^{228} \mathrm{Ac}$, the magnetic moment of ${ }^{226} \mathrm{Ac}$ corresponds to empirical values deduced using the additivity rule and the experimental magnetic moment of ${ }^{227} \mathrm{Ac}$ and ${ }^{225} \mathrm{Ra}$. The latter are explained 
TABLE VI. Empirical magnetic dipole moments for ${ }^{226} \mathrm{Ac}$ obtained by coupling the proton states of ${ }^{225,227} \mathrm{Ac}(I=3 / 2)$ with the neutron state of ${ }^{225} \mathrm{Ra}(I=1 / 2)$. Compare with the experimental value $\mu_{\exp }\left({ }^{226} \mathrm{Ac}\right)=1.06(0.04)\{0.03\} \mu_{N}$.

\begin{tabular}{lccc}
\hline \hline \multirow{2}{*}{$\begin{array}{l}\text { Proton } \\
\text { state }\end{array}$} & $\begin{array}{c}\text { Neutron } \\
\text { state }\end{array}$ & \multicolumn{2}{c}{$\mu_{\text {emp }}\left(\mu_{N}\right)$} \\
\cline { 3 - 4 } & & $g_{s}=0.6 g_{s}^{\text {free }}$ & $g_{s}=0.8 g_{s}^{\text {free }}$ \\
\hline${ }^{227} \mathrm{Ac}$ & ${ }^{225} \mathrm{Ra}$ & $1.255(0.015)\{0.025\}$ & $1.159(0.015)\{0.025\}$ \\
${ }^{225} \mathrm{Ac}$ & ${ }^{225} \mathrm{Ra}$ & $1.347(0.016)\{0.033\}$ & $1.251(0.016)\{0.033\}$ \\
\hline \hline
\end{tabular}

in the framework of a single-particle approach with a nonzero octupole deformation (see Sec. III E 1 and Refs. [10,101]) and thus hint to the presence of octupole deformation in ${ }^{226} \mathrm{Ac}$.

\section{CONCLUSIONS AND OUTLOOK}

The hyperfine spectra of isotopes ${ }^{225-229}$ Ac have been measured using in-source resonance ionization laser spectroscopy at the ISAC facility in TRIUMF, probing a ${ }^{2} D_{3 / 2} \rightarrow$ ${ }^{4} P_{5 / 2}^{\circ}$ atomic transition. From the measured hyperfine spectra, data on the changes in the nuclear mean-square charge radii and the magnetic dipole moments of isotopes ${ }^{226,228,229} \mathrm{Ac}$ have been extracted. Comparison with the high-resolution data set [28] for isotopes ${ }^{225,227}$ Ac allowed for systematicuncertainty assignments on the deduced $\delta\left\langle r^{2}\right\rangle^{A, 215}$ and magnetic dipole moment values of the newly investigated isotopes.

Two conclusions could be drawn about the mean-square charge radii of the actinium isotopes. First, the charge radii follow the same trend as previously observed in the neighboring radium and francium isotopes on both the neutron-rich and neutron-deficient edges of the nuclear chart. Second, the charge radii display an inverted odd-even staggering for ${ }^{226} \mathrm{Ac}$, while the relative odd-even staggering parameter for ${ }^{228} \mathrm{Ac}$ is within the large experimental uncertainties consistent with zero.

Calculations with three different nuclear EDFs have been performed differing in their form, fit strategy and capacity to describe charge radii around ${ }^{208} \mathrm{~Pb}$ : SLy5s1, BSk31, and DDMEB1. For SLy5s1 in particular, fully fledged self-consistent blocked HFB calculations of odd-odd nuclei simultaneously considering reflection and time-reversal symmetry breaking are reported for the first time. The explanation of the spectroscopic features of ${ }^{225-229}$ Ac implies a large static intrinsic octupole deformation of these nuclei. Our calculations find octupole-deformed ground states for actinium isotopes with $130<N<142$. It is shown that the description of the experimental $\delta\left\langle r^{2}\right\rangle$ values of the actinium isotopes with $N>136$ is markedly better when the octupole degree of freedom is taken into account.

The magnetic dipole moments of the odd-mass actinium isotopes are compared with results from an octupole-deformed Wood-Saxon shell-model rotor-plusparticle approach [10] as well as with values obtained in the framework of the reflection-symmetrical Nilsson model [6]. These comparisons indicate a noticeable octupole deformation in ${ }^{225,227}$ Ac with decreasing importance in ${ }^{229} \mathrm{Ac}$. These conclusions are further supported by comparing the magnetic dipole moments of ${ }^{226,228}$ Ac with predictions using the additivity rule.

It will be instructive to extend as well as to repeat the actinium laser spectroscopy measurements using highresolution methods such as the in-gas-jet technique previously applied for the neutron-deficient actinium isotopes [27]. This would not only allow for reducing the uncertainties on the mean-square charge radii and magnetic dipole moments of the isotopes ${ }^{226,228,229} \mathrm{Ac}$ and determining their quadrupole moments but also for extending the investigated neutron-rich actinium region toward ${ }^{222-234} \mathrm{Ac}$, where octupole deformation is expected to disappear (see Fig. 4 in Ref. [27]).

\section{ACKNOWLEDGMENTS}

This work has been funded by FWO-Vlaanderen (Belgium), by GOA/2015/010 (BOF KU Leuven), by the Interuniversity Attraction Poles Programme initiated by the Belgian Science Policy Offce (BriX network P7/12), by the European Commission within the Seventh Framework Programme through I3-ENSAR (Contract No. RII3-CT-2010262010) and by a grant from the European Research Council (Grant No. ERC-2011-AdG-291561-HELIOS), and by the European Union's Horizon 2020 research and innovation programme under Grant Agreement No. 654002. The work was supported by RFBR according to the research Project No. N 19-02-00005. The MOCCa computations were performed using HPC resources from the Consortium des Équipements de Calcul Intensif (CÉCI), funded by the Fonds de la Recherche Scientifique de Belgique (F.R.S.-FNRS) under Grant No. 2.5020.11, and the computing center of the IN2P3/CNRS. TRIUMF's core operations are supported via a contribution from the federal government through the National Research Council Canada (NRC). J.L. acknowledges additional support through Natural Sciences and Engineering Research Council of Canada (NSERC) discovery Grants No. GP-386343-2011 and No. SAP-IN-2017-00039. We acknowledge the support of W. Gins (KU Leuven) for guidance with his open-source python package SATLAS.
[1] P. A. Butler and W. Nazarewicz, Rev. Mod. Phys. 68, 349 (1996).

[2] B. Bucher, S. Zhu, C. Y. Wu, R. V. F. Janssens, D. Cline, A. B. Hayes, M. Albers, A. D. Ayangeakaa, P. A. Butler, C. M. Campbell, M. P. Carpenter, C. J. Chiara, J. A. Clark, H. L. Crawford, M. Cromaz, H. M. David,
C. Dickerson, E. T. Gregor, J. Harker, C. R. Hoffman, B. P. Kay, F. G. Kondev, A. Korichi, T. Lauritsen, A. O. Macchiavelli, R. C. Pardo, A. Richard, M. A. Riley, G. Savard, M. Scheck, D. Seweryniak, M. K. Smith, R. Vondrasek, and A. Wiens, Phys. Rev. Lett. 116, 112503 (2016). 
[3] V. Spevak, N. Auerbach, and V. V. Flambaum, Phys. Rev. C 56, 1357 (1997).

[4] P. Möller and J. R. Nix, Nucl. Phys. A 361, 117 (1981).

[5] W. Nazarewicz, P. Olanders, and I. Ragnarsson, Nucl. Phys. A 429, 269 (1984).

[6] G. Leander and R. Sheline, Nucl. Phys. A 413, 375 (1984).

[7] R. K. Sheline, Phys. Lett. B 197, 500 (1987).

[8] R. K. Sheline, Phys. Rev. C 37, 423 (1988).

[9] G. A. Leander, R. K. Sheline, P. Möller, P. Olanders, I. Ragnarsson, and A. J. Sierk, Nucl. Phys. A 388, 452 (1982).

[10] G. A. Leander and Y. S. Chen, Phys. Rev. C 37, 2744 (1988).

[11] P. Bonche, P.-H. Heenen, H. Flocard, and D. Vautherin, Phys. Lett. B 175, 387 (1986).

[12] L. M. Robledo, J. L. Egido, J. F. Berger, and M. Girod, Phys. Lett. B 187, 223 (1987)

[13] K. Rutz, J. A. Maruhn, P.-G. Reinhard, and W. Greiner, Nucl. Phys. A 590, 680 (1995).

[14] S. E. Agbemava, A. V. Afanasjev, and P. Ring, Phys. Rev. C 93, 044304 (2016).

[15] Z. P. Li, B. Y. Song, J. M. Yao, D. Vretenar, and J. Meng, Phys. Lett. B 726, 866 (2013).

[16] L. M. Robledo and R. R. Rodríguez-Guzmán, J. Phys. G: Nucl. Part. Phys. 39, 105103 (2012).

[17] L. M. Robledo and G. F. Bertsch, Phys. Rev. C 84, 054302 (2011).

[18] K. Nomura, D. Vretenar, and B.-N. Lu, Phys. Rev. C 88, 021303(R) (2013).

[19] E. W. Otten, Treatise on Heavy Ion Science (Springer US, Boston, MA, 1989), Sec. 4, pp. 517-638.

[20] W. Borchers, R. Neugart, E. W. Otten, H. T. Duong, G. Ulm, and K. Wendt, Hyperfine Interact. 34, 25 (1987).

[21] V. A. Dzuba, W. R. Johnson, and M. S. Safronova, Phys. Rev. A 72, 022503 (2005).

[22] I. Budinčević, J. Billowes, M. L. Bissell, T. E. Cocolios, R. P. de Groote, S. De Schepper, V. N. Fedosseev, K. T. Flanagan, S. Franchoo, R. F. Garcia Ruiz, H. Heylen, K. M. Lynch, B. A. Marsh, G. Neyens, T. J. Procter, R. E. Rossel, S. Rothe, I. Strashnov, H. H. Stroke, and K. D. A. Wendt, Phys. Rev. C 90, 014317 (2014)

[23] L. W. Wansbeek, S. Schlesser, B. K. Sahoo, A. E. L. Dieperink, C. J. G. Onderwater, and R. G. E. Timmermans, Phys. Rev. C 86, 015503 (2012).

[24] I. Angeli and M. Csatlós, Nucl. Phys. A 288, 480 (1977).

[25] F. Buchinger, J. E. Crawford, A. K. Dutta, J. M. Pearson, and F. Tondeur, Phys. Rev. C 49, 1402 (1994).

[26] A. Teigelhöfer, Ph.D. thesis, University of Manitoba, 2017.

[27] R. Ferrer, A. Barzakh, B. Bastin, R. Beerwerth, M. Block, P. Creemers, H. Grawe, R. de Groote, P. Delahaye, X. Fléchard, S. Franchoo, S. Fritzsche, L. P. Gaffney, L. Ghys, W. Gins, C. Granados, R. Heinke, L. Hijazi, M. Huyse, T. Kron, Y. Kudryavtsev, M. Laatiaoui, N. Lecesne, M. Loiselet, F. Lutton, I. D. Moore, Y. Martínez, E. Mogilevskiy, P. Naubereit, J. Piot, S. Raeder, S. Rothe, H. Savajols, S. Sels, V. Sonnenschein, J.-C. Thomas, E. Traykov, C. Van Beveren, P. Van den Bergh, P. Van Duppen, K. Wendt, and A. Zadvornaya, Nat. Commun. 8, 14520 (2017).

[28] S. Reader, R. Beerwerth, M. Block, P. Chhetri, R. Ferrer, S. Fritsche, F. Giacoppo, C. Granados, A. Hakimi, R. Heinke, T. Kron, M. Laatiaoui, V. Sonnenschein, H. Tomita, P. Van Duppen, E. Verstraelen, and K. Wendt (unpublished).
[29] C. Granados Ph.D. thesis, KU Leuven (unpublished).

[30] C. Granados, P. Creemers, R. Ferrer, L. P. Gaffney, W. Gins, R. de Groote, M. Huyse, Y. Kudryavtsev, Y. Martínez, S. Raeder, S. Sels, C. Van Beveren, P. Van den Bergh, P. Van Duppen, K. Wrzosek-Lipska, A. Zadvornaya, A. E. Barzakh, B. Bastin, P. Delahaye, L. Hijazi, N. Lecesne, F. Luton, J. Piot, H. Savajols, J.-C. Thomas, E. Traykov, R. Beerwerth, S. Fritzsche, M. Block, X. Fléchard, S. Franchoo, L. Ghys, H. Grawe, R. Heinke, T. Kron, P. Naubereit, K. Wendt, M. Laatiaoui, I. Moore, V. Sonnenschein, M. Loiselet, E. Mogilevskiy, and S. Rothe, Phys. Rev. C 96, 054331 (2017).

[31] J. Roßnagel, S. Raeder, A. Hakimi, R. Ferrer, N. Trautmann, and K. Wendt, Phys. Rev. A 85, 012525 (2012).

[32] S. Raeder, B. Bastin, M. Block, P. Creemers, P. Delahaye, R. Ferrer, X. Fléchard, S. Franchoo, L. Ghys, L. P. Gaffneya et al., Nucl. Instrum. Methods B 376, 382 (2016).

[33] R. Heinke, T. Kron, S. Raeder, T. Reich, P. Schönberg, M. Trümper, C. Weichhold, and K. Wendt, Hyperfine Interact. 238 (2017).

[34] J. Lassen, R. Li, S. Raeder, X. Zhao, T. Dekker, H. Heggen, P. Kunz, C. D. P. Levy, M. Mostanmand, A. Teigelhöfer, and F. Ames, Hyperfine Interact. 238, 33 (2017).

[35] J. Dilling, R. Krücken, and G. Ball, Hyperfine Interact. 225, 1 (2014).

[36] P. Kunz, P. Bricault, M. Dombsky, N. Erdmann, V. Hanemaayer, J. Wong, and K. Lützenkirchen, J. Nucl. Mater. 440, 110 (2013).

[37] V. Sonnenschein, Ph.D. thesis, University of Jyväskylä, 2015.

[38] P. Kunz, C. Andreoiu, P. Bricault, M. Dombsky, J. Lassen, A. Teigelhöfer, H. Heggen, and F. Wong, Rev. Sci. Instrum. 85, 053305 (2014).

[39] P. Campbell, I. D. Moore, and M. R. Pearson, Prog. Part. Nucl. Phys. 86, 127 (2016).

[40] W. Gins, R. P. de Groote, M. L. Bissell, C. Granados Buitrago, R. Ferrer, K. M. Lynch, G. Neyens, and S. Sels, Comput. Phys. Commun. 222, 286 (2018).

[41] J. Persson, At. Data Nucl. Data Tables 99, 62 (2013).

[42] S. K. Bhattacherjee, S. K. Mitra, H. C. Jain, H. C. Padhi, K. S. Bhatki, and D. C. Ephraim, Nucl. Phys. A 90, 696 (1967).

[43] Y. A. Akovali, Nucl. Data Sheets 77, 433 (1996).

[44] E. Browne and J. K. Tuli, Nucl. Data Sheets 109, 2657 (2008).

[45] M. Wang, G. Audi, F. G. Kondev, W. Huang, S. Naimi, and X. Xu, Chin. Phys. C 41, 030003 (2017).

[46] D. Berdichevsky and F. Tondeur, Z. Phys. A 322, 141 (1985).

[47] M. Anselment, W. Faubel, S. Göring, A. Hanser, G. Meisel, H. Rebel, and G. Schatz, Nucl. Phys. A 451, 471 (1986).

[48] A. E. Barzakh, D. V. Fedorov, V. S. Ivanov, P. L. Molkanov, F. V. Moroz, S. Y. Orlov, V. N. Panteleev, M. D. Seliverstov, and Y. M. Volkov, Phys. Rev. C 95, 044324 (2017).

[49] M. R. Pearson, P. Campbell, K. Leerungnavarat, J. Billowes, I. S. Grant, M. Keim, J. Kilgallon, I. D. Moore, R. Neugart, M. Neuroth, S. Wilbert, and The ISOLDE Collaboration, J. Phys. G: Nucl. Part. Phys. 26, 1829 (2000).

[50] A. E. Barzakh, D. V. Fedorov, V. S. Ivanov, P. L. Molkanov, F. V. Moroz, S. Y. Orlov, V. N. Panteleev, M. D. Seliverstov, and Y. M. Volkov, Phys. Rev. C 97, 014322 (2018).

[51] T. E. Cocolios, W. Dexters, M. D. Seliverstov, A. N. Andreyev, S. Antalic, A. E. Barzakh, B. Bastin, J. Büscher, I. G. Darby, D. V. Fedorov, V. N. Fedosseyev, K. T. Flanagan, S. Franchoo, S. Fritzsche, G. Huber, M. Huyse, M. Keupers, U. Köster, 
Y. Kudryavtsev, E. Mané, B. A. Marsh, P. L. Molkanov, R. D. Page, A. M. Sjoedin, I. Stefan, J. Van de Walle, P. Van Duppen, M. Venhart, S. G. Zemlyanoy, M. Bender, and P.-H. Heenen, Phys. Rev. Lett. 106, 052503 (2011).

[52] D. A. Fink, T. E. Cocolios, A. N. Andreyev, S. Antalic, A. E. Barzakh, B. Bastin, D. V. Fedorov, V. N. Fedosseev, K. T. Flanagan, L. Ghys, A. Gottberg, M. Huyse, N. Imai, T. Kron, N. Lecesne, K. M. Lynch, B. A. Marsh, D. Pauwels, E. Rapisarda, S. D. Richter, R. E. Rossel, S. Rothe, M. D. Seliverstov, A. M. Sjödin, C. Van Beveren, P. Van Duppen, and K. D. A. Wendt, Phys. Rev. X 5, 011018 (2015).

[53] M. D. Seliverstov, T. E. Cocolios, W. Dexters, A. N. Andreyev, S. Antalic, A. E. Barzakh, B. Bastin, J. Büscher, I. G. Darby, D. V. Fedorov, V. N. Fedoseyev, K. T. Flanagan, S. Franchoo, S. Fritzsche, G. Huber, M. Huyse, M. Keupers, U. Köster, Y. Kudryavtsev, B. A. Marsh, P. L. Molkanov, R. D. Page, A. M. Sjødin, I. Stefan, J. Van de Walle, P. Van Duppen, M. Venhart, and S. G. Zemlyanoy, Phys. Lett. B 719, 362 (2013).

[54] D. Kowalewska, K. Bekk, S. Göring, A. Hanser, W. Kälber, G. Meisel, and H. Rebel, Phys. Rev. A 44, R1442 (1991).

[55] J. G. Cubiss, A. E. Barzakh, M. D. Seliverstov, A. N. Andreyev, B. Andel, S. Antalic, P. Ascher, D. Atanasov, D. Beck, J. Bieroń, K. Blaum, C. Borgmann, M. Breitenfeldt, L. Capponi, T. E. Cocolios, T. Day Goodacre, X. Derkx, H. De Witte, J. Elseviers, D. V. Fedorov, V. N. Fedosseev, S. Fritzsche, L. P. Gaffney, S. George, L. Ghys, F. P. Heßberger, M. Huyse, N. Imai, Z. Kalaninová, D. Kisler, U. Köster, M. Kowalska, S. Kreim, J. F. W. Lane, V. Liberati, D. Lunney, K. M. Lynch, V. Manea, B. A. Marsh, S. Mitsuoka, P. L. Molkanov, Y. Nagame, D. Neidherr, K. Nishio, S. Ota, D. Pauwels, L. Popescu, D. Radulov, E. Rapisarda, J. P. Revill, M. Rosenbusch, R. E. Rossel, S. Rothe, K. Sandhu, L. Schweikhard, S. Sels, V. L. Truesdale, C. Van Beveren, P. Van den Bergh, Y. Wakabayashi, P. Van Duppen, K. D. A. Wendt, F. Wienholtz, B. W. Whitmore, G. L. Wilson, R. N. Wolf, and K. Zuber, Phys. Rev. C 97, 054327 (2018).

[56] A. E. Barzakh, J. G. Cubiss, A. N. Andreyev, M. D. Seliverstov, B. Andel, S. Antalic, P. Ascher, D. Atanasov, D. Beck, J. Bieroń, K. Blaum, C. Borgmann, M. Breitenfeldt, L. Capponi, T. E. Cocolios, T. Day Goodacre, X. Derkx, H. De Witte, J. Elseviers, D. V. Fedorov, V. N. Fedosseev, S. Fritzsche, L. P. Gaffney, S. George, L. Ghys, F. P. Heßberger, M. Huyse, N. Imai, Z. Kalaninová, D. Kisler, U. Köster, M. Kowalska, S. Kreim, J. F. W. Lane, V. Liberati, D. Lunney, K. M. Lynch, V. Manea, B. A. Marsh, S. Mitsuoka, P. L. Molkanov, Y. Nagame, D. Neidherr, K. Nishio, S. Ota, D. Pauwels, L. Popescu, D. Radulov, E. Rapisarda, J. P. Revill, M. Rosenbusch, R. E. Rossel, S. Rothe, K. Sandhu, L. Schweikhard, S. Sels, V. L. Truesdale, C. Van Beveren, P. Van den Bergh, P. Van Duppen, Y. Wakabayashi, K. D. A. Wendt, F. Wienholtz, B. W. Whitmore, G. L. Wilson, R. N. Wolf, and K. Zuber, Phys. Rev. C 99, 054317 (2019).

[57] G. J. Farooq-Smith, T. E. Cocolios, J. Billowes, M. L. Bissell, I. Budinčević, T. Day Goodacre, R. P. de Groote, V. N. Fedosseev, K. T. Flanagan, S. Franchoo, R. F. Garcia Ruiz, H. Heylen, R. Li, K. M. Lynch, B. A. Marsh, G. Neyens, R. E. Rossel, S. Rothe, H. H. Stroke, K. D. A. Wendt, S. G. Wilkins, and X. F. Yang, Phys. Rev. C 94, 054305 (2016).

[58] K. M. Lynch, S. G. Wilkins, J. Billowes, C. L. Binnersley, M. L. Bissell, K. Chrysalidis, T. E. Cocolios, T. D. Goodacre, R. P. de Groote, G. J. Farooq-Smith, D. V. Fedorov, V. N.
Fedosseev, K. T. Flanagan, S. Franchoo, R. F. Garcia Ruiz, W. Gins, R. Heinke, Á. Koszorús, B. A. Marsh, P. L. Molkanov, P. Naubereit, G. Neyens, C. M. Ricketts, S. Rothe, C. Seiffert, M. D. Seliverstov, H. H. Stroke, D. Studer, A. R. Vernon, K. D. A. Wendt, and X. F. Yang, Phys. Rev. C 97, 024309 (2018).

[59] W. J. Tomlinson and H. H. Stroke, Nucl. Phys. 60, 614 (1964).

[60] S. A. Ahmad, W. Klempt, R. Neugart, E. W. Otten, P.-G. Reinhard, G. Ulm, and K. Wendt, Nucl. Phys. A 483, 244 (1988).

[61] S. A. Fayans, S. V. Tolokonnikov, E. L. Trykov, and D. Zawischa, Nucl. Phys. A 676, 49 (2000).

[62] M. Hammen, W. Nörtershäuser, D. L. Balabanski, M. L. Bissell, K. Blaum, I. Budinčević, B. Cheal, K. T. Flanagan, N. Frömmgen, G. Georgiev, C. Geppert, M. Kowalska, K. Kreim, A. Krieger, W. Nazarewicz, R. Neugart, G. Neyens, J. Papuga, P.-G. Reinhard, M. M. Rajabali, S. Schmidt, and D. T. Yordanov, Phys. Rev. Lett. 121, 102501 (2018).

[63] P.-G. Reinhard and W. Nazarewicz, Phys. Rev. C 95, 064328 (2017).

[64] P. Lievens, E. Arnold, W. Borchers, U. Georg, M. Keim, A. Klein, R. Neugart, L. Vermeeren, and R. E. Silverans, Europhys. Lett. 33, 11 (1996).

[65] B. A. Marsh, T. Day Goodacre, S. Sels, Y. Tsunoda, B. Andel, A. N. Andreyev, N. A. Althubiti, D. Atanasov, A. E. Barzakh, J. Billowes, K. Blaum, T. E. Cocolios, J. G. Cubiss, J. Dobaczewski, G. J. Farooq-Smith, D. V. Fedorov, V. N. Fedosseev, K. T. Flanagan, L. P. Gaffney, L. Ghys, M. Huyse, S. Kreim, D. Lunney, K. M. Lynch, V. Manea, Y. Martinez Palenzuela, P. L. Molkanov, T. Otsuka, A. Pastore, M. Rosenbusch, R. E. Rossel, S. Rothe, L. Schweikhard, M. D. Seliverstov, P. Spagnoletti, C. Van Beveren, P. Van Duppen, M. Veinhard, E. Verstraelen, A. Welker, K. Wendt, F. Wienholtz, R. N. Wolf, A. Zadvornaya, and K. Zuber, Nat. Phys. 14, 1163 (2018).

[66] W. D. Myers and K.-H. Schmidt, Nucl. Phys. A 410, 61 (1983).

[67] H. Iimura and F. Buchinger, Phys. Rev. C 78, 067301 (2008).

[68] H. Iimura, P. Möller, T. Ichikawa, H. Sagawa, and A. Iwamoto, JPS Conf. Proc. 6, 030102 (2015).

[69] W. Ryssens, M. Bender, K. Bennaceur, P.-H. Heenen, and J. Meyer, Phys. Rev. C 99, 044315 (2019).

[70] J. Dobaczewski and J. Engel, Phys. Rev. Lett. 94, 232502 (2005).

[71] J. Dobaczewski, J. Engel, M. Kortelainen, and P. Becker, Phys. Rev. Lett. 121, 232501 (2018).

[72] D. Peña-Arteaga, S. Goriely, and N. Chamel, Eur. Phys. J. A 52, 320 (2016).

[73] S. Goriely, N. Chamel, and J. M. Pearson, Phys. Rev. C 93, 034337 (2016).

[74] R. Jodon, M. Bender, K. Bennaceur, and J. Meyer, Phys. Rev. C 94, 024335 (2016).

[75] G. Audi, M. Wang, A. H. Wapstra, F. G. Kondev, M. MacCormick, X. Xu, and B. Pfeiffer, Chin. Phys. C 36, 1603 (2012).

[76] I. Angeli, At. Data Nucl. Data Tables 87, 185 (2004).

[77] R. Niembro, S. Marcos, M. López-Quelle, and L. N. Savushkin, Phys. At. Nucl. 75, 269 (2012).

[78] M. M. Sharma, G. A. Lalazissis, and P. Ring, Phys. Lett. B 317, 9 (1993). 
[79] P.-G. Reinhard and H. Flocard, Nucl. Phys. A 584, 467 (1995).

[80] M. M. Sharma, G. Lalazissis, J. König, and P. Ring, Phys. Rev. Lett. 74, 3744 (1995).

[81] J.-P. Ebran, A. Mutschler, E. Khan, and D. Vretenar, Phys. Rev. C 94, 024304 (2016).

[82] S. Goriely, Nucl. Phys. A 933, 68 (2015).

[83] S. Perez-Martin and L. M. Robledo, Phys. Rev. C 78, 014304 (2008).

[84] W. Ryssens, Ph.D. thesis, Université Libre de Bruxelles, 2016.

[85] W. Ryssens, M. Bender, and P. H. Heenen, Eur. Phys. J. A 55, 93 (2019)

[86] J. W. Negele, Phys. Rev. C 1, 1260 (1970).

[87] W. Ryssens, V. Hellemans, M. Bender, and P.-H. Heenen, Comput. Phys. Commun. 187, 175 (2015).

[88] L. Vermeeren, R. E. Silverans, P. Lievens, A. Klein, R. Neugart, C. Schulz, and F. Buchinger, Phys. Rev. Lett. 68, 1679 (1992).

[89] R. F. Garcia Ruiz, M. L. Bissell, K. Blaum, A. Ekström, N. Frömmgen, G. Hagen, M. Hammen, K. Hebeler, J. D. Holt, G. R. Jansen, M. Kowalska, K. Kreim, W. Nazarewicz, R. Neugart, G. Neyens, W. Nörtershäuser, T. Papenbrock, J. Papuga, A. Schwenk, J. Simonis, K. A. Wendt, and D. T. Yordanov, Nat. Phys. 12, 594 (2016).

[90] C. Gorges, L. V. Rodríguez, D. L. Balabanski, M. L. Bissell, K. Blaum, B. Cheal, R. F. Garcia Ruiz, G. Georgiev, W. Gins, H. Heylen, A. Kanellakopoulos, S. Kaufmann, M. Kowalska,
V. Lagaki, S. Lechner, B. Maaß, S. Malbrunot-Ettenauer, W. Nazarewicz, R. Neugart, G. Neyens, W. Nörtershäuser, P.-G. Reinhard, S. Sailer, R. Sánchez, S. Schmidt, L. Wehner, C. Wraith, L. Xie, Z. Y. Xu, X. F. Yang, and D. T. Yordanov, Phys. Rev. Lett. 122, 192502 (2019).

[91] W. Ryssens and M. Bender (unpublished).

[92] J. Zhang, M. Tandecki, R. Collister, S. Aubin, J. A. Behr, E. Gomez, G. Gwinner, L. A. Orozco, M. R. Pearson, and G. D. Sprouse, Phys. Rev. Lett. 115, 042501 (2015).

[93] C. Ekström, Phys. Scr. 13, 217 (1976).

[94] R. Neugart and G. Neyens, in The Euroschool Lectures on Physics with Exotic Beams, Vol. II, edited by J. Al-Khalili and E. Roeckl, Lecture Notes in Physics Vol. 700 (Springer, Berlin, 2006), pp. 135-189.

[95] I. L. Lamm, Nucl. Phys., Sect. A 125, 504 (1969).

[96] C. Ekström, L. Robertsson, and A. Rosén, Phys. Scr. 34, 624 (1986).

[97] R. K. Sheline and G. A. Leander, Phys. Rev. Lett. 51, 359 (1983).

[98] Nuclear Data Center website (2017), https://www.nndc.bnl. gov/.

[99] P. Möller, A. J. Sierk, T. Ichikawa, and H. Sagawa, At. Data Nucl. Data Tables 109-110, 1 (2016).

[100] S. Ahmad, W. Klempt, R. Neugart, E. W. Otten, K. Wendt, and C. Ekström, Phys. Lett. B 133, 47 (1983).

[101] I. Ragnarsson, Phys. Lett. B 130, 353 (1983). 